\title{
FORMAL CONSTRUCTIONS IN THE BRAUER GROUP OF THE FUNCTION FIELD OF A $p$-ADIC CURVE
}

\author{
ERIC BRUSSEL AND EDUARDO TENGAN
}

\begin{abstract}
We study the relationship between the cohomology of the function field of a curve over a complete discretely valued field and that of the function ring of curves resulting over its residue field. The results are applied to prove the existence of noncrossed product division algebras and indecomposable division algebras of unequal period and index over the function field of any $p$-adic curve, generalizing the results and methods of a previous work of the authors and McKinnie.
\end{abstract}

\section{INTRODUCTION}

Let $F$ be a field. An $F$-division algebra $D$ is a division ring that is finitedimensional over $F$ and whose center is $F$. We say $D$ is a crossed product if $D$ contains a Galois field extension $E / F$ that is maximal in $D$ with respect to containment of subfields, and a noncrossed product if it does not. $D$ is a crossed product precisely when its multiplication rule can be described by a Galois 2-cocycle with values in the multiplicative group $E^{*}$ of a Galois extension $E / F$, and as a result all early division algebra constructions were crossed products by default. The classical fact that every $F$-division algebra contains a separable maximal subfield implies that the matrix algebra $\mathrm{M}_{n}(D)$ is a crossed product for some $n$, for any $D$. During the development of class field theory it was established that all $\mathbb{Q}$ and $\mathbb{Q}_{p}$-division algebras are crossed products via a cyclic Galois extension. Decades later, noncrossed product $F$-division algebras were shown to exist, in a 1972 paper by Amitsur ([1]). Since then several different and interesting constructions have appeared over various fields (see [5, Section 0] for additional background).

An $F$-division algebra is decomposable if it can be expressed as an $F$-tensor product of two (nontrivial) F-division algebras, and indecomposable otherwise. Division algebras admit two measures of size called period and index, analogous to exponent and order for finite abelian groups. Before it was proved otherwise, it would have been reasonable to conjecture that any $F$-division algebra of unequal period and index would decompose into factors of equal period and index. Indecomposable division algebras of unequal period and index first appeared in 1979, in papers by Amitsur-Rowen-Tignol [3] and Saltman [37. Since then there have been several other constructions, notably by Jacob in [25] and Karpenko in [26, 27] (see [5. Section 9]).

Received by the editors March 21, 2012 and, in revised form, April 11, 2013.

2010 Mathematics Subject Classification. Primary 11G20, 11R58, 14E22, 16K50.

The second author was supported by CNPq grant 303817/2011-9. 
$F$-division algebras are fundamentally arithmetic objects in the sense that they become trivial when scalar-extended to an algebraic closure of $F$. As such their taxonomy is a reflection of $F$ 's arithmetic, and this motivates our interest in constructions of noncrossed products and indecomposables of unequal period and index. We are particularly interested in the relationship between possible constructions over arithmetically related fields, such as the function fields of curves over discretely valued fields and over their residue fields.

The Brauer group $\operatorname{Br}(F)$ is a group formed by isomorphism classes of $F$-division algebras, and its $n$-torsion subgroup is isomorphic (via the crossed product construction) to the degree-two cohomology group $\mathrm{H}^{2}\left(F, \mu_{n}\right)$, for $n$ prime-to-char $(F)$. In this paper we study the cohomology groups $\mathrm{H}^{q}(F,(\mathbb{Z} / n)(i))$ of a field $F$ that is finitely generated and of transcendence degree one over a complete discretely valued field $K=(K, v)$, and in particular over the $p$-adic field $\mathbb{Q}_{p}$. Such a field $F$ is always the function field of a regular, projective, flat relative curve $X / \mathrm{O}_{v}$. In [10] it was shown that if $K=\mathbb{Q}_{p}$ and $F$ admits a smooth model $X / \mathbb{Z}_{p}$, then there exist noncrossed product $F$-division algebras, and indecomposable $F$-division algebras of unequal prime-power period and index. This applies to fields such as $\mathbb{Q}_{p}(t)$ but not to the function fields of all $p$-adic curves. In this paper we generalize the machinery of [10] to the function field $F$ of an arbitrary $p$-adic curve, and then prove the existence of noncrossed product $F$-division algebras, and indecomposable $F$-division algebras of unequal prime-power period and index.

The Brauer groups of function fields of $p$-adic curves have been the focus of several important papers recently, including the work by Saltman 39, 41, which initiated much of the recent activity, work by Harbater-Hartmann-Krashen [23, 24], which uses patching methods, and work by Lieblich [30] and Parimala-Suresh 34 ] (on the $u$-invariant of quadratic forms). The use of 2-dimensional models to study these Brauer groups goes back at least to Saito [36] and Kato 28], and ultimately to Grothendieck.

Our technique is to lift constructions from the rational function ring $\kappa(C)$ of the reduced scheme $C$ underlying the closed fiber of $X / \mathbb{Z}_{p}$, which is a product of global fields. Our main technical advance over [10] is to show how to do this if $C$ is reducible with mild singularities. This situation can be unavoidable, and arises naturally after blowups of $X$. The extra generality has crucial implications for the theory of $\operatorname{Br}(F)$, and allows us, for example, to prove in [11] that every $F$-division algebra of prime period $\ell \neq p$ and index $\ell^{2}$ decomposes into two cyclic $F$-tensor factors, hence is a crossed product, even when $F=\mathbb{Q}_{p}(t)$. The latter result generalizes Suresh's result [42, which assumes roots of unity, and does not cover $\mathbb{Q}_{p}(t)$ in general.

We summarize the technical results. Let $K=(K, v)$ be a complete discretely valued field $K$ with residue field $k, n$ a number prime-to-char $(k)$, and let $F / K$ be a finitely generated field extension of transcendence degree one. Let $X / \mathrm{O}_{v}$ be a regular, projective, flat, 2-dimensional model for $F$. The closed fiber $X_{0} / k$ is a projective curve, whose underlying reduced structure $C$ may be assumed to consist of regular irreducible components $C_{i}$, exactly two of which meet at the singular points $\mathcal{S}$ of $C$. Our main theorem (Theorem 4.9) constructs for any integer $r$ and any $q \geq 0$ a homomorphism

$$
\lambda: \mathrm{H}^{q}\left(\mathrm{O}_{C, \mathcal{S}}, \mathbb{Z} / n(r)\right) \longrightarrow \mathrm{H}^{q}\left(\mathrm{O}_{X, \mathcal{S}}, \mathbb{Z} / n(r)\right) \hookrightarrow \mathrm{H}^{q}(F, \mathbb{Z} / n(r))
$$

whose first arrow splits the restriction map $\mathrm{H}^{q}\left(\mathrm{O}_{X, \mathcal{S}}, \mathbb{Z} / n(r)\right) \rightarrow \mathrm{H}^{q}\left(\mathrm{O}_{C, \mathcal{S}}, \mathbb{Z} / n(r)\right)$. 
Let $F_{C}=\prod_{i} F_{C_{i}}$ be the product of the completions of $F$ with respect to the valuations defined by the $C_{i}$. We use $\lambda$ to construct for any $q \geq 1$ a commutative diagram

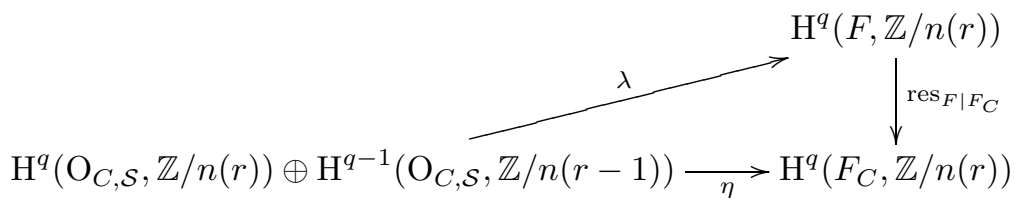

so that $\lambda\left(\left(\alpha_{C}, \theta_{C}\right)\right)=\lambda\left(\alpha_{C}\right)+(\pi) \cdot \lambda\left(\theta_{C}\right)$ for a uniformizer $\pi$ of $F_{C}$. When $K=\mathbb{Q}_{p}$, $q=2$, and $r=1$, we show that $\lambda$ factors through $\mathrm{H}^{2}\left(F_{C}, \mu_{n}\right)$ to determine an indexpreserving map from the subgroup $\operatorname{im}(\eta) \leq \mathrm{H}^{2}\left(F_{C}, \mu_{n}\right)$ to $\mathrm{H}^{2}\left(F, \mu_{n}\right)$, splitting the restriction map. Since the residue fields $\kappa\left(C_{i}\right)$ in this case are global fields we are then able to construct indecomposable $F$-division algebras and noncrossed product $F$-division algebras, in the same manner as 10 . When the dual graph of $C$ has nontrivial topology, i.e., nonzero (first) Betti number, we construct cyclic covers of $X$ that are (defined and) trivial at every point of $X$ except the generic point of $X$. These arise as cyclic covers of $C$ that are trivial at every point, and transported to $X$ via $\lambda$. When $K=\mathbb{Q}_{p}$ they are the completely split cyclic covers of Saito ([36]). We thank Colliot-Thélène for drawing our attention to these interesting examples.

\section{BACKGROUND AND CONVENTIONS}

We use [31, Chapter 8,9], 22, Section 2], and [21, Chapter XIII] for many of the following definitions.

2.1. General conventions. Let $S$ be an excellent scheme, $n$ a number that is invertible on $S$, and for any $i \in \mathbb{Z}$ let $\Lambda=(\mathbb{Z} / n)(i)$ be the twisted étale sheaf. Let $K(S)$ denote the total fraction ring of $S$, which is the ring of global sections of the sheaf of total fractions. We write $\mathrm{H}^{q}(S, \Lambda)$ for the étale cohomology group, and if $\Lambda$ is understood (or doesn't matter) we write $\mathrm{H}^{q}(S, r)$ instead of $\mathrm{H}^{q}(S, \Lambda(r)$ ), and $\mathrm{H}^{q}(S)$ in place of $\mathrm{H}^{q}(S, 0)$. If $S=\operatorname{Spec} A$ for a ring $A$, then we write $\mathrm{H}^{q}(A, r)$. If $D$ is a closed subscheme of $S$ we write $\kappa(D)$ for its total fraction ring. If $T \rightarrow S$ is a morphism of schemes, then the restriction $\operatorname{res}_{S \mid T}: \mathrm{H}^{q}(S) \rightarrow \mathrm{H}^{q}(T)$ is defined, and we write $\beta_{T}=\operatorname{res}_{S \mid T}(\beta)$. If $S=\operatorname{Spec} A$ and $T=\operatorname{Spec} B$ for a $\operatorname{ring} B$ we write $\beta_{B}=\operatorname{res}_{A \mid B}(\beta)$ instead. If $Z \rightarrow S$ is another morphism, we write $Z_{T}$ for the fiber product $Z \times{ }_{S} T$.

2.2. Valuation theory. If $v$ is a discrete valuation on a field $F$ we write $\kappa(v)$ for the residue field of the valuation ring $\mathrm{O}_{v}$, and $F_{v}$ for the completion of $F$ at $v$. If $S$ is a noetherian connected normal scheme with function field $F$ and $v$ arises from a prime divisor $D$ on $S$, we generally substitute $D$ for $v$, and write $v_{D}$ for $v, \kappa(D)$ for $\kappa(v)$, and $F_{D}$ for $F_{v}$. If $D$ is a sum of prime divisors $D_{i}$ we write $F_{D}=\prod_{i} F_{D_{i}}$. We will abuse this notation and write $F_{D}$ should $D$ be a closed subscheme of pure codimension one.

By "Witt's Theorem" (see [16, 7.10]) for each discrete valuation $v$ on the field $F$ there is a short exact sequence

$$
0 \longrightarrow \mathrm{H}^{q}(\kappa(v), \Lambda) \stackrel{\mathrm{inf}}{\longrightarrow} \mathrm{H}^{q}\left(F_{v}, \Lambda\right) \stackrel{\partial_{v}}{\longrightarrow} \mathrm{H}^{q-1}(\kappa(v), \Lambda(-1)) \longrightarrow 0
$$

where inf is the inflation map and $\partial_{v}$ is the residue map. We use this sequence to identify $\mathrm{H}^{q}(\kappa(v), \Lambda)$ with the corresponding subgroup of $\mathrm{H}^{q}\left(F_{v}, \Lambda\right)$. If 
$\alpha \in \mathrm{H}^{q}\left(F_{v}, \Lambda\right)$, then $\partial_{v}(\alpha)$ is called the residue of $\alpha$ at $v$. More generally, suppose $T$ is a noetherian scheme, $\xi$ is a generic point of $T$, and $\alpha \in \mathrm{H}^{q}(T, \Lambda)$. Then for each discrete valuation $v$ on the field $F=\kappa(\xi), \alpha$ has a residue

$$
\partial_{v}(\alpha) \stackrel{\text { df }}{=} \partial_{v}\left(\alpha_{F_{v}}\right) \in \mathrm{H}^{q-1}(\kappa(v), \Lambda(-1)) .
$$

We say $\alpha$ is unramified at $v$ if $\partial_{v}(\alpha)=0$ and ramified at $v$ if $\partial_{v}(\alpha) \neq 0$. If $\alpha$ is unramified at $v$ the value of $\alpha$ at $v$ is the element

$$
\alpha(v)=\alpha_{F_{v}} \in \mathrm{H}^{q}(\kappa(v), \Lambda) \leq \mathrm{H}^{q}\left(F_{v}, \Lambda\right)
$$

(see [16, 7.13, p.19]). Suppose $T$ is as above and $T \rightarrow S$ is a birational morphism of noetherian schemes (see [17, I.2.2.9]). The ramification locus of $\alpha \in \mathrm{H}^{q}(T, \Lambda)$ on $S_{\text {red }}$ is the sum (disregarding multiplicities) of the prime divisors on $S_{\text {red }}$ that determine valuations at which $\alpha$ is ramified, over all generic points of $S_{\text {red }}$.

2.3. Tamely ramified covers. Let $S$ be a noetherian normal scheme, $D$ a divisor on $S, U=S-D$, and for each generic point $\xi$ of $\operatorname{Supp} D$, let $K_{\xi}=$ Frac $_{S, \xi}$. Note that $S$ being normal implies that the irreducible components of $S$ are disjoint. We say $\rho: T \rightarrow(S, D)$ is a tamely ramified cover (ramified along $D$ ) if

(1) $\rho_{U}: V=T \times{ }_{S} U \rightarrow U$ is étale;

(2) $\rho: T \rightarrow S$ is finite;

(3) each irreducible component of $T$ dominates an irreducible component of $S$;

(4) $T$ is normal;

(5) for each generic point $\xi$ of $\operatorname{Supp} D$, the étale $K_{\xi}$-algebra $L$ defined by Spec $L=V \times_{U}$ Spec $K_{\xi}$ is tamely ramified with respect to the discrete valuation determined by $\xi$.

In (5) $L / K_{\xi}$ is étale, hence $L$ is a finite product of separable field extensions of $K_{\xi}$, and $L$ is tamely ramified if each field extension is tamely ramified (with respect to $\mathrm{O}_{S, \xi}$ ) in the usual sense. If $S=\operatorname{Spec} A$ and $T=\operatorname{Spec} B$, we will also say $B$ is a finite tamely ramified A-algebra. This is the definition [22, Definition 2.2.2] given by Grothendieck-Murre. If $S$ is regular and $D$ has normal crossings on $S$, the last condition (5) is equivalent to the definition [21, XIII.2.3(c)] given in SGA 1, allowing us to use the results of [21, XIII.5].

If $S$ is a noetherian scheme whose irreducible components are normal, we will say $\rho: T \rightarrow(S, D)$ is a tamely ramified cover (ramified along $D$ ) if again $\rho$ is finite and $V \rightarrow U=S-D$ is étale, and the restriction $\rho_{S_{i}}$ to each irreducible component $S_{i}$ of $S$ is a tamely ramified cover (ramified along $D_{S_{i}}$ ).

2.4. Standard setup. $K=(K, v)$ is a complete discretely valued field with complete discrete valuation ring $R=\mathrm{O}_{v}$ and residue field $k, F / K$ is a finitely generated field extension of transcendence degree, and $X$ is a regular connected 2 -dimensional scheme that is flat and projective over $\operatorname{Spec} R$ and has function field $F=K(X)$. We call $X / R$ a (regular) relative curve, and write $X_{0}=X \otimes_{R} k$ for the closed fiber (a projective curve over $k$ by [31, 8.3.3]), $C=X_{0, \text { red }}$ for the reduced subscheme underlying $X_{0}$, and $C_{1}, \ldots, C_{m}$ for the irreducible components of $C$. We assume each $C_{i}$ is regular, and at most two of them meet at any closed point of $X$, a situation that can always be obtained by blowing up, using Lipman's embedded resolution theorem (see [31, 9.2.4]). We let $\mathcal{S}$ denote the set of singular points of $C$. Note that all closed points of $X$ lie on $C$, and for each closed point $z \in X$ we have $\operatorname{dim} \mathrm{O}_{X, z}=2$ by [31, 8.3.4(c)]. Since $X$ is regular, $\mathrm{O}_{X, z}$ is then factorial by Auslander-Buchsbaum's theorem. 
We say an effective (Cartier) divisor $D$ on our relative curve $X / R$ is horizontal if each irreducible component of $\operatorname{Supp} D$ maps surjectively (hence finitely by 31 , 8.3.4(a),(b)]) to $\operatorname{Spec} R$, and vertical if $\operatorname{Supp} D$ is contained in $C$. If $D$ is a reduced and irreducible horizontal divisor, then it is flat over $\operatorname{Spec} R$, since $R$ is a discrete valuation ring. Every effective divisor on a regular relative curve $X / R$ is a sum of horizontal and vertical divisors, and the horizontal prime divisors are exactly the closures of the closed points of the generic fiber ([31, 8.3.4(b)]). Since $R$ is henselian, each irreducible horizontal divisor has a single closed point. More generally, since $R$ is henselian and $X / R$ is projective there is a 1-1 correspondence between connected components of a horizontal divisor $D$ and connected components of $D \otimes_{R} k$ ([19, Proposition 18.5.19]).

2.5. Distinguished divisors. In general there will be many horizontal divisors on our $X / R$ that restrict to a given divisor on $C$. In order to construct our lifts from $C$ to $X$ we select a single regular horizontal divisor for each closed point, as follows.

Proposition 2.6. Assume the setup of 2.4. Then for each closed point $z \in X$ there exists a regular irreducible effective horizontal divisor $D \subset X$ that intersects each irreducible component of $C$ passing through $z$ transversally at $z$.

Proof. Transversal intersection with a single component is by [31, 8.3.35(b)] and its proof (see also [20, 21.9.12]). Thus if $z \in C_{i} \cap C_{j}(i \neq j)$ and $t_{i}$ and $t_{j}$ are local equations for $C_{i}$ and $C_{j}$, then we have local equations $f_{i}$ and $f_{j}$ for effective regular horizontal divisors such that $\left(f_{i}, t_{i}\right)=\left(f_{j}, t_{j}\right)=\mathfrak{m}_{z} \subset \mathrm{O}_{X, z}$. If $\left(f_{j}, t_{i}\right)=\mathfrak{m}_{z}$ or $\left(f_{i}, t_{j}\right)=\mathfrak{m}_{z}$, then a suitable $D$ is defined locally by $f_{j}$ or $f_{i}$. Otherwise $\left(f_{i}+f_{j}, t_{i}\right)=\left(f_{i}+f_{j}, t_{j}\right)=\mathfrak{m}_{z}$, and we define $D$ locally by $f_{i}+f_{j}$. The rest of the proof proceeds as in [31, 8.3.35].

We fix a set of these (prime) divisors, and let $\mathscr{D}$ denote the union of their supports. We will say a divisor $D$ is distinguished and write $D \in \mathscr{D}$ whenever $D$ is reduced and supported in $\mathscr{D}$. Let $\mathscr{D}_{\mathcal{S}}$ denote the subset that avoids the set of singular points $\mathcal{S}$ of $C$. Note that each $D \in \mathscr{D}$ is a disjoint union of its irreducible components, each of which meets each irreducible component of $C$ transversally.

\section{Structure of tame COVERS}

Lemma 3.1 (Structure). Assume the setup of 2.4. Suppose $\rho: Y \rightarrow(X, D)$ is a tamely ramified cover, where $D \in \mathscr{D}$. Then:

a) The structure map $\rho: Y \rightarrow X$ is flat.

b) $Y / R$ is a regular relative curve, $Y_{0, \text { red }}=C_{Y}$, each irreducible component of $C_{Y}$ is regular, $\mathcal{S}_{Y}=\rho^{-1}(\mathcal{S})$ is the set of singular closed points of $C_{Y}$, and exactly two irreducible components of $C_{Y}$ meet at each point of $\mathcal{S}_{Y}$.

c) The support of the irreducible components of $D_{Y}^{\prime}$ for $D^{\prime} \in \mathscr{D}$ generate a set $\mathscr{D}_{Y}$ of distinguished divisors on $Y$.

Proof. Since $Y \rightarrow X$ is finite, $\operatorname{dim}(X)=\operatorname{dim}(Y)=2$ by [31, 2.5.10], and $Y \rightarrow$ Spec $R$ is projective as the composition of projective morphisms ([31, 3.3.32]). Let $y \in Y$ be a closed point and set $x=f(y), A=\mathrm{O}_{X, x}, B^{\prime}=\mathrm{O}_{Y, x}$, and $B=\mathrm{O}_{Y, y}$. By $B^{\prime}$ we mean the ring of the fiber over $\operatorname{Spec}_{X, x}$ on $Y$. Choose a geometric point over $x$ that lifts to each point of $Y$ lying over $x$, and use this in the following to define the strict henselizations with respect to the maximal ideals of these points. 
Since the statements involving $D$ are local and $D$ is a disjoint union of its irreducible components we may assume $D$ is irreducible. Let $C_{i} \subset C$ be a (regular) irreducible component going through $x$, and let $\{f, t\} \subset A$ be the regular system of parameters formed by local equations for the distinguished prime divisor passing through $x$, and for $C_{i}$, respectively. Then the strict henselization $A^{\text {sh }}$ of $A$ with respect to the maximal ideal of $A$ is a 2-dimensional regular local ring, faithfully flat over $A$, with regular system of parameters $\{f, t\}$ (see [20, 18.8]).

If $x \notin D$, then $B^{\prime} \otimes_{A} A^{\text {sh }}$ is a finite étale $A^{\text {sh }}$-algebra by base change, since $\left.\rho\right|_{X-D}$ is finite-étale. If $x \in D$, then $B^{\prime} \otimes_{A} A^{\text {sh }}$ is a finite tamely ramified $A^{\text {sh }}$-algebra by [22, Lemma 2.2.8]. By [20, 18.8.10], $B^{\text {sh }}$ is a factor of the direct product decomposition of $B^{\prime} \otimes_{A} A^{\text {sh }}$, hence $B^{\text {sh }}$ is a finite tamely ramified local $A^{\text {sh }}$-algebra, in particular it is a normal local ring, hence it is a normal domain. It follows that $B^{\text {sh }}$ is the integral closure of $A^{\text {sh }}$ in the field $\widetilde{L} \stackrel{\text { df }}{=}$ Frac $B^{\text {sh }}$. Since the tame fundamental group of the strictly henselian regular local ring $A^{\text {sh }}$ is abelian ([21, XIII.5.3]) the field extension $\widetilde{L} /$ Frac $A^{\text {sh }}$ is Galois, and by Abhyankar's Lemma ([13, A.I.11], see also [22, Corollary 2.3.4])

$$
B^{\mathrm{sh}}=A^{\mathrm{sh}}[T] /\left(T^{e}-f\right) \quad(\text { some } e \geq 1) .
$$

By [22, Lemma 1.8.6] $B^{\text {sh }}$ is a regular (2-dimensional) local ring with system of parameters $\{\sqrt[e]{f}, t\}$. Since $B \rightarrow B^{\text {sh }}$ is faithfully flat and $B^{\text {sh }}$ is regular, $B$ is regular by flat descent $([18,6.5 .1]$ or $[32,23.7(\mathrm{i})])$, and since $B$ is the local ring of an arbitrary closed point, we conclude $Y$ is regular. It follows that $\rho: Y \rightarrow X$ is flat by [32, 23.1], proving (a), and since $Y$ is regular and $Y \rightarrow \operatorname{Spec} R$ is flat and projective, $Y / R$ is a regular relative curve.

We derive a system of parameters for $B$. The prime ideal $(\sqrt[e]{f}) \subset B^{\text {sh }}$ is the only one lying over $(f) A^{\text {sh }}$ since, for $\kappa(f)=$ Frac $A^{\text {sh }} /(f) A^{\text {sh }}$, the ring $B^{\text {sh }} \otimes_{A^{\text {sh }}} \kappa(f)=$ $\kappa(f)[T] /\left(T^{e}\right)$ of the fiber over Spec $\kappa(f)$ consists of a single prime ideal. Since $\operatorname{Spec} B^{\text {sh }} \rightarrow$ Spec $B$ is surjective, the image $(\sqrt[e]{f}) \cap B$ of $(\sqrt[e]{f})$ in Spec $B$ is the unique prime lying over $(f) \subset A$, and since $B \rightarrow B^{\text {sh }}$ is flat, $(\sqrt[e]{f}) \cap B$ has height one, hence is principal (since $B$ is factorial), hence $(\sqrt[e]{f}) \cap B=(g)$ for some $g \in B$. Then $(\sqrt[e]{f})$ is the unique prime of $B^{\text {sh }}$ lying over $(g)$, and $(g)$ is the unique prime of $B$ lying over $(f)$. Since $B \rightarrow B^{\text {sh }}$ is unramified, $(g) B^{\text {sh }}=(\sqrt[e]{f})$. Since $B \rightarrow B^{\text {sh }}$ is faithfully flat, $I B^{\mathrm{sh}} \cap B=I$ for all ideals $I$ of $B$ (by e.g. [4, Exercise 3.16]), so since $(g, t) B^{\mathrm{sh}}=(\sqrt[e]{f}, t)$ is maximal, $(g, t) B^{\mathrm{sh}} \cap B=(g, t)$ is the maximal ideal of $B$. Thus $\{g, t\}$ is a regular system for $B$.

Since $t$ is a local equation for $\rho^{-1} C_{i}, \rho^{-1} C_{i}$ is regular and irreducible at $y$ for each $C_{i}$ passing through $x$. In particular $C_{Y}=\bigcup_{i} \rho^{-1} C_{i}$ is reduced, and so equals $Y_{0, \text { red }}$, and since at most two irreducible components of $C$ meet at $x$, at most two irreducible components of $C_{Y}$ meet at $y$. Since $\operatorname{Spec} B \rightarrow \operatorname{Spec} A$ is surjective, $C_{Y}$ has at least the number of components at $y$ as does $C$ at $x$. Thus $y$ is a singular point on $C_{Y}$ if and only if $x=f(y) \in \mathcal{S}$. This completes the proof of (b).

If $D^{\prime} \in \mathscr{D}$ is the distinguished (horizontal) prime divisor running through $x$, then there is a single irreducible component of $D_{Y}^{\prime}$ passing through $y$, whose support $D_{Y \text {,red }}^{\prime}$ has local equation $g$ at $y$. Thus each irreducible component of $D_{Y}^{\prime}$ covers $D^{\prime}$, hence Spec $R$, hence $D_{Y}^{\prime}$ is horizontal. Since $g$ is part of the regular system $\{g, t\}$ at the arbitrary closed point $y$ we see that $D_{Y, \text { red }}^{\prime}$ is regular, and since $t$ is a local equation for an arbitrary irreducible component of $C_{Y}$ passing through $y, D_{Y \text {,red }}^{\prime}$ intersects each component of $C_{Y}$ transversally. Thus the supports of the irreducible 
components of the various $D_{Y}^{\prime}$ for $D^{\prime} \in \mathscr{D}$ generate a set of distinguished divisors $\mathscr{D}_{Y}$ for $Y$. This proves (c).

Lemma 3.2. Suppose $X$ is a regular noetherian scheme and $L$ is an étale $K(X)$ algebra that is tamely ramified along a divisor $D$. Then the normalization $Y$ of $X$ in $L$ defines a tamely ramified cover $\rho: Y \rightarrow(X, D)$.

Proof. Since $X$ is regular, its connected components are integral regular schemes, hence we may assume $X$ is integral. Since $L / K(X)$ is étale, $L$ is a product of finite separable field extensions of $K(X)$, hence we may assume $L / K(X)$ is itself a finite separable field extension. Then the normalization $Y$ exists, $Y$ is normal by definition, and $\rho: Y \rightarrow X$ is finite by [31, 4.1.25]. Since $\rho: Y \rightarrow X$ induces an injection $K(X) \rightarrow K(Y), Y$ dominates $X$. Let $U=X-D$, and set $V=Y \times_{X} U$. Since $X$ is normal, $Y$ is connected, and $\left.\rho\right|_{V}$ is unramified, $\left.\rho\right|_{V}$ is étale by [21, I.9.11] (see also [33, I.3.20]). Therefore $Y \rightarrow(X, D)$ is a tamely ramified cover.

The next lemma shows how distinguished divisors split in tamely ramified covers.

Lemma 3.3. Assume the setup of 2.4. Suppose $\rho: Y \rightarrow(X, D)$ is a tamely ramified cover, where $D \in \mathscr{D}$, and $D^{\prime} \in \mathscr{D}_{\mathcal{S}}$ is irreducible. Suppose $E \subset D_{Y \text {,red }}^{\prime}$ is a distinguished prime divisor lying over $D^{\prime}$ as in Lemma 3.1) (c), $y=E \times_{Y} C_{Y}$, and $x=D^{\prime} \times_{X} C$. Then $y$ and $x$ are regular closed points, and the ramification (resp. inertia) degree of $v_{E}$ over $v_{D^{\prime}}$ equals the ramification (resp. inertia) degree of $v_{y}$ over $v_{x}$.

Proof. Since we assume 2.4 and $D \in \mathscr{D}$ we have Lemma 3.1, which shows $C_{Y}$ is reduced and $E \subset D_{Y, \text { red }}^{\prime}$ is distinguished. Note that either $D^{\prime} \cap D=\varnothing$ or $D^{\prime} \subset D$. Since $D^{\prime}$ and $E$ are distinguished and avoid the singular points of $C$ and $C_{Y}$, they intersect the reduced closed fibers $C$ and $C_{Y}$ transversally, hence $x=D^{\prime} \times_{X} C$ and $y=E \times_{Y} C_{Y}$ are regular closed points. We must show that $\left[\kappa(E): \kappa\left(D^{\prime}\right)\right]=[\kappa(y): \kappa(x)]$ and that $v_{E}(f)=v_{y}\left(f_{0}\right)$, where $f \in \mathrm{O}_{X, D^{\prime}}$ is a local equation for $D^{\prime}$ on $X$ and $f_{0} \in \mathrm{O}_{C, x}$ is a local equation for $x$ on $C$.

Since $D^{\prime}$ is horizontal and irreducible, $D^{\prime}=\operatorname{Spec} S$ for $S$ a finite local $R$-algebra by [31, 8.3.4] and [33, I.4.2], and $S$ is a discrete valuation ring since $D^{\prime}$ is regular. The map $E \rightarrow \rho^{-1} D^{\prime} \rightarrow D^{\prime}$ is finite as a composition of finite morphisms, hence $E=\operatorname{Spec} T$ for $T$ a finite local $S$-algebra, again a discrete valuation ring since $E$ is regular. Since $S$ is a discrete valuation ring, $S \rightarrow T$ is finite, and $T$ is torsionfree, $T$ is a free $S$-module of finite rank, and so $[T: S]$ is well defined. Since the generic point of $E$ lies over that of $D^{\prime}$, we have Frac $T=T \otimes_{S}$ Frac $S$, hence $\left[\kappa(E): \kappa\left(D^{\prime}\right)\right]=[T: S]$.

Let $A=\mathrm{O}_{X, x}, B=\mathrm{O}_{Y, y}$, let $t$ be a local equation for $C$ at $x$, and set $A_{0}=A /(t)$ and $B_{0}=B /(t) B$, the (reduced) local rings of the fibers through $x$ and $y$, as in the proof of Lemma 3.1. Already $\kappa(x)=S \otimes_{A} A_{0}$ and $\kappa(y)=T \otimes_{B} B_{0}$ by the transversality of the intersections. Since $B_{0}=B \otimes_{A} A_{0}$ we have $\kappa(y)=T \otimes_{A} A_{0}$, hence $[\kappa(y): \kappa(x)]=[T: S]=\left[\kappa(E): \kappa\left(D^{\prime}\right)\right]$ by base change.

Let $f \in A$ and $g \in B$ be defined as in the proof of Lemma 3.1. To compute the ramification degree, note that since $B \rightarrow B^{\text {sh }}$ is faithfully flat, $\left(g^{e}\right) B=\left(g^{e}\right) B^{\text {sh }} \cap$ $B=(f) B^{\text {sh }} \cap B=(f) B$, hence $g^{e}=f u$ for some $u \in B^{*}$. Since $f$ and $g$ are uniformizers for $v_{D^{\prime}}$ and $v_{E}$, respectively, it follows that $e\left(v_{E} / v_{D^{\prime}}\right)=v_{E}(f)=e$. On the other hand, let $f_{0}$ be the image of $f$ in $A_{0}$, and let $g_{0}$ be the image of $g$ in $B_{0}$. Then $f_{0}$ cuts out the closed point $x$ on $C$ and $g_{0}$ cuts out $y$ on $C_{Y}$ by 
transversality. Thus $f_{0}$ and $g_{0}$ are uniformizers for $v_{x}$ and $v_{y}$, and since $g_{0}^{e}=f_{0} u_{0}$, where $u_{0}$ is the image of $u$ in $B_{0}^{*}$, we have $e\left(v_{y} / v_{x}\right)=v_{y}\left(f_{0}\right)=e$, as desired. This completes the proof.

\section{Lifting COHOMOLOGY CLASSES}

4.1. Let $k$ be a field, and let $C / k$ be a reduced connected projective curve with regular irreducible components $C_{1}, \ldots, C_{m}$, at most two of which meet at any closed point. Denote the singular points of $C$ by $\mathcal{S}$ and write $\mathrm{O}_{C, \mathcal{S}}$ for the semilocal ring $\lim _{U} \mathrm{O}_{C}(U)$, where $U$ varies over (dense) open subsets of $C$ containing $\mathcal{S}$. Then since $C$ has no embedded points, $\mathrm{O}_{C, \mathcal{S}}$ is a subring of the rational function ring $\kappa(C)=\prod_{i} \kappa\left(C_{i}\right)$ by [31, 7.1.9]. For each $z \in \mathcal{S} \cap C_{i}$, let $K_{i, z}=$ Frac $\mathrm{O}_{C_{i}, z}^{\mathrm{h}}$, a field since $z$ is a normal point of $C_{i}$, and if $\alpha_{i} \in \mathrm{H}^{q}\left(\kappa\left(C_{i}\right)\right)$, let $\alpha_{i, z}$ denote the image in $\mathrm{H}^{q}\left(K_{i, z}\right)$.

Since $C / k$ is projective it is separated (over $\mathbb{Z}$ ), hence if $U \subset C$ is an affine open subset of $C$, then $U \rightarrow C$ is an affine map by [31, 3.3.6]. The inverse limit $\lim U$ over affine open subschemes of $C$ containing $\mathcal{S}$ is then a scheme by [19, 8.2.3], and this scheme is $\operatorname{Spec}_{C, \mathcal{S}}$ by [31, Exercise 5.1.17(c)]. Therefore $\mathrm{H}^{q}\left(\mathrm{O}_{C, \mathcal{S}}, \Lambda\right)=$ $\underset{\lim }{\longrightarrow} \mathrm{H}^{q}(U, \Lambda)$ by [33, III.1.16].

Lemma 4.2 (Gluing). Assume the setup of 4.1. There exists an element $\alpha \in$ $\mathrm{H}^{q}\left(\mathrm{O}_{C, \mathcal{S}}, \Lambda\right)$ that restricts to $\alpha_{C}=\left(\alpha_{1}, \ldots, \alpha_{m}\right) \in \bigoplus_{i} \mathrm{H}^{q}\left(\kappa\left(C_{i}\right), \Lambda\right)$ if and only if $\alpha_{i}$ is unramified at each $z \in \mathcal{S} \cap C_{i}$, and $\alpha_{i, z}=\alpha_{j, z}$ (as elements of $\mathrm{H}^{q}(\kappa(z), \Lambda)$ ) whenever $z \in C_{i} \cap C_{j}$.

Proof. There is an exact sequence ([33, III.1.25])

$$
\begin{aligned}
0 \longrightarrow \mathrm{H}_{\mathcal{S}}^{0}\left(\mathrm{O}_{C, \mathcal{S}}\right) \longrightarrow & \mathrm{H}^{0}\left(\mathrm{O}_{C, \mathcal{S}}\right) \longrightarrow \mathrm{H}^{0}(\kappa(C)) \longrightarrow \mathrm{H}_{\mathcal{S}}^{1}\left(\mathrm{O}_{C, \mathcal{S}}\right) \longrightarrow \\
& \longrightarrow \mathrm{H}^{1}\left(\mathrm{O}_{C, \mathcal{S}}\right) \longrightarrow \mathrm{H}^{1}(\kappa(C)) \longrightarrow \mathrm{H}_{\mathcal{S}}^{2}\left(\mathrm{O}_{C, \mathcal{S}}\right) \longrightarrow \\
& \longrightarrow \mathrm{H}^{2}\left(\mathrm{O}_{C, \mathcal{S}}\right) \longrightarrow \mathrm{H}^{2}(\kappa(C)) \longrightarrow \mathrm{H}_{\mathcal{S}}^{3}\left(\mathrm{O}_{C, \mathcal{S}}\right) \longrightarrow
\end{aligned}
$$

where the maps $\mathrm{H}^{q}\left(\mathrm{O}_{C, \mathcal{S}}\right) \rightarrow \mathrm{H}^{q}(\kappa(C))$ are restrictions. Since $\mathcal{S}$ is a disjoint union of closed points, we have $\mathrm{H}_{\mathcal{S}}^{q}\left(\mathrm{O}_{C, \mathcal{S}}\right)=\bigoplus_{z \in \mathcal{S}} \mathrm{H}_{z}^{q}\left(\mathrm{O}_{C, \mathcal{S}}\right)=\bigoplus_{z \in \mathcal{S}} \mathrm{H}_{z}^{q}\left(\mathrm{O}_{C, z}^{\mathrm{h}}\right)$ by excision ([33. III.1.28, p.93]). Since $\Lambda$ is a smooth group scheme, $\mathrm{H}^{q}\left(\mathrm{O}_{C, z}^{\mathrm{h}}\right)=\mathrm{H}^{q}(\kappa(z))$, by the cohomological Hensel's lemma [33, III.3.11(a), p.116]. Since the $C_{k}$ are regular and exactly two of them meet at any $z \in \mathcal{S}$, we have $\operatorname{Spec}_{C, z}^{\mathrm{h}}-\{z\}=$ $\operatorname{Spec}\left(K_{i, z} \times K_{j, z}\right)$ for some $i$ and $j$, and an "excised" exact sequence

$$
\begin{aligned}
0 \longrightarrow \mathrm{H}_{z}^{0}\left(\mathrm{O}_{C, z}^{\mathrm{h}}\right) \longrightarrow & \mathrm{H}^{0}(\kappa(z)) \longrightarrow \mathrm{H}^{0}\left(K_{i, z} \times K_{j, z}\right) \longrightarrow \mathrm{H}_{z}^{1}\left(\mathrm{O}_{C, z}^{\mathrm{h}}\right) \longrightarrow \\
& \longrightarrow \mathrm{H}^{1}(\kappa(z)) \longrightarrow \mathrm{H}^{1}\left(K_{i, z} \times K_{j, z}\right) \longrightarrow \mathrm{H}_{z}^{2}\left(\mathrm{O}_{C, z}^{\mathrm{h}}\right) \longrightarrow \\
& \longrightarrow \mathrm{H}^{2}(\kappa(z)) \longrightarrow \mathrm{H}^{2}\left(K_{i, z} \times K_{j, z}\right) \longrightarrow \mathrm{H}_{z}^{3}\left(\mathrm{O}_{C, z}^{\mathrm{h}}\right) \longrightarrow
\end{aligned}
$$

where the map $\mathrm{H}^{q}(\kappa(z)) \rightarrow \mathrm{H}^{q}\left(K_{i, z} \times K_{j, z}\right)=\mathrm{H}^{q}\left(K_{i, z}\right) \oplus \mathrm{H}^{q}\left(K_{j, z}\right)$ is the diagonal map given by inflation from $\kappa(z)$ to the henselian fields $K_{i, z}$ and $K_{j, z}$. Since $n$ is prime-to- $p$, the map $\mathrm{H}^{0}(\kappa(z)) \rightarrow \mathrm{H}^{0}\left(K_{i, z}\right)$ is an isomorphism, so $\mathrm{H}_{z}^{0}\left(\mathrm{O}_{C, z}^{\mathrm{h}}\right)=0$, and for $q \geq 1$ we have short exact Witt-type sequences

$$
0 \rightarrow \mathrm{H}^{q}(\kappa(z)) \rightarrow \mathrm{H}^{q}\left(K_{i, z}\right) \stackrel{\partial_{z}}{\longrightarrow} \mathrm{H}^{q-1}(\kappa(z),-1) \rightarrow 0 .
$$

Thus the long exact sequence breaks up into short exact sequences

$$
0 \rightarrow \mathrm{H}^{q}(\kappa(z)) \rightarrow \mathrm{H}^{q}\left(K_{i, z} \times K_{j, z}\right) \rightarrow \mathrm{H}_{z}^{q+1}\left(\mathrm{O}_{C, z}^{\mathrm{h}}\right) \rightarrow 0 \quad(q \geq 0) .
$$


By the compatibility of (4.3) with (4.4) the map $\mathrm{H}^{q}\left(\kappa\left(C_{i}\right)\right) \rightarrow \mathrm{H}_{z}^{q+1}\left(\mathrm{O}_{C, z}^{\mathrm{h}}\right) \leq$ $\mathrm{H}_{\mathcal{S}}^{q+1}\left(\mathrm{O}_{C, \mathcal{S}}\right)$ factors through $\operatorname{res}_{\kappa\left(C_{i}\right) \mid K_{i, z}}$. Therefore an element $\alpha_{C}=\left(\alpha_{1}, \ldots, \alpha_{m}\right)$ $\in \mathrm{H}^{q}(\kappa(C))$ maps to zero in $\mathrm{H}_{\mathcal{S}}^{q+1}\left(\mathrm{O}_{C, \mathcal{S}}\right)$ if and only if each couple $\left(\alpha_{i, z}, \alpha_{j, z}\right)$ is in the image of some $\bar{\alpha} \in \mathrm{H}^{q}(\kappa(z))$; i.e., $\alpha_{i, z}=\alpha_{j, z}$, and both are unramified. Thus by the exactness of (4.3),$\alpha_{C}$ comes from $\mathrm{H}^{q}\left(\mathrm{O}_{C, \mathcal{S}}\right)$ if and only if each $\alpha_{i}$ is unramified at each $z \in \mathcal{S} \cap C_{i}$, and $\alpha_{i, z}=\alpha_{j, z}$ whenever $z \in C_{i} \cap C_{j}$.

Suppose $C$ is as in 4.1. Since exactly two irreducible components meet at any $z \in \mathcal{S}$ the dual graph $G_{C}$ is defined, and consists of a vertex for each irreducible component of $C$ and an edge for each singular point, such that an edge and a vertex are incident when the corresponding singular point lies on the corresponding irreducible component ([36, 2.23]; see also [31, 10.1.48]). The (first) Betti number for $G_{C}$ is $\beta_{C} \stackrel{\text { df }}{=} \operatorname{rk}\left(\mathrm{H}_{1}\left(G_{C}, \mathbb{Z}\right)\right)=N+E-V$, where $V, E$ and $N$ are the numbers of vertices, edges, and connected components of $G_{C}$, respectively. Note $N=1$ in the setup of 4.1 .

Lemma 4.6. Assume the setup of 4.1. Then:

a) For any integer $r, \mathrm{H}^{1}(C, \mathbb{Z} / n(r)) \rightarrow \mathrm{H}^{1}\left(\mathrm{O}_{C, \mathcal{S}}, \mathbb{Z} / n(r)\right)$ is injective.

b) The map $\mathrm{H}^{q}\left(\mathrm{O}_{C, \mathcal{S}}, \mathbb{Z} / n(q-1)\right) \rightarrow \mathrm{H}^{q}(\kappa(C), \mathbb{Z} / n(q-1))$ is injective for $q=0,2$, and for $q=1$ we have

$$
\mathrm{H}^{1}\left(\mathrm{O}_{C, \mathcal{S}}, \mathbb{Z} / n\right) \simeq(\mathbb{Z} / n)^{\beta_{C}} \oplus \Gamma
$$

where $(\mathbb{Z} / n)^{\beta_{C}}$ is the kernel of $\mathrm{H}^{1}\left(\mathrm{O}_{C, \mathcal{S}}, \mathbb{Z} / n\right) \rightarrow \mathrm{H}^{1}(\kappa(C), \mathbb{Z} / n)$, and $\Gamma \leq$ $\mathrm{H}^{1}(\kappa(C), \mathbb{Z} / n)$ is the group of tuples that glue as in Lemma 4.2 .

Proof. We suppress the notation for $\Lambda=\mathbb{Z} / n(r)$. Let $\mathcal{F} \subset C-\mathcal{S}$ be a finite set of (regular) closed points, and set $U=C-\mathcal{F}$, a dense open subset containing $\mathcal{S}$. The localization exact sequence is

$$
\begin{aligned}
0 \longrightarrow \mathrm{H}_{\mathcal{F}}^{0}(C) & \longrightarrow \mathrm{H}^{0}(C) \longrightarrow \mathrm{H}^{0}(U) \longrightarrow \cdots \\
\cdots & \longrightarrow \mathrm{H}_{\mathcal{F}}^{q}(C) \longrightarrow \mathrm{H}^{q}(C) \longrightarrow \mathrm{H}^{q}(U) \longrightarrow \mathrm{H}_{\mathcal{F}}^{q+1}(C) \longrightarrow \cdots
\end{aligned}
$$

By excision we have an exact sequence

$$
\begin{aligned}
0 \longrightarrow \mathrm{H}_{\mathcal{F}}^{0}(C) \longrightarrow \bigoplus_{z \in \mathcal{F}} \mathrm{H}^{0}\left(\mathrm{O}_{C, z}^{\mathrm{h}}\right) \longrightarrow \bigoplus_{z \in \mathcal{F}} \mathrm{H}^{0}\left(K_{z}\right) \longrightarrow \cdots \\
\cdots \longrightarrow \mathrm{H}_{\mathcal{F}}^{q}(C) \longrightarrow \bigoplus_{z \in \mathcal{F}} \mathrm{H}^{q}\left(\mathrm{O}_{C, z}^{\mathrm{h}}\right) \longrightarrow \bigoplus_{z \in \mathcal{F}} \mathrm{H}^{q}\left(K_{z}\right) \longrightarrow \mathrm{H}_{\mathcal{F}}^{q+1}(C) \longrightarrow \cdots
\end{aligned}
$$

where $K_{z}=$ Frac $\mathrm{O}_{C, z}^{\mathrm{h}}$. Since each $z$ is a regular point, $\mathrm{O}_{C, z}^{\mathrm{h}}$ is a discrete valuation ring, and by [12, Section 3.6] we may replace each $\mathrm{H}_{z}^{q+1}(C)$ with $\mathrm{H}^{q-1}(\kappa(z),-1)$, and the map from $\mathrm{H}^{q}(U)$, which factors through each $\mathrm{H}^{q}\left(K_{z}\right)$, is then the residue map $\partial_{z}$. We conclude $\mathrm{H}^{0}(C)=\mathrm{H}^{0}(U)$, and we have a long exact sequence

$$
\begin{aligned}
0 \longrightarrow \mathrm{H}^{1}(C) \longrightarrow \mathrm{H}^{1}(U) \stackrel{\partial_{z}}{\longrightarrow} \bigoplus_{z \in \mathcal{F}} \mathrm{H}^{0}(\kappa(z),-1) \longrightarrow \cdots \\
\cdots \longrightarrow \mathrm{H}^{q}(C) \longrightarrow \mathrm{H}^{q}(U) \stackrel{\partial_{z}}{\longrightarrow} \bigoplus_{z \in \mathcal{F}} \mathrm{H}^{q-1}(\kappa(z),-1) \longrightarrow \cdots
\end{aligned}
$$

As $\mathrm{H}^{1}\left(\mathrm{O}_{C, \mathcal{S}}\right)=\lim _{U} \mathrm{H}^{1}(U)$, where the limit is over all such $U, \mathrm{H}^{1}(C) \rightarrow \mathrm{H}^{1}\left(\mathrm{O}_{C, \mathcal{S}}\right)$ is injective by the exactness of the injective limit functor, proving (a). 
For (b) we go back to $\Lambda=\mathbb{Z} / n$. By (4.3) we have an exact sequence

$$
\begin{aligned}
& 0 \longrightarrow \mathrm{H}^{0}\left(\mathrm{O}_{C, \mathcal{S}}, \mathbb{Z} / n\right) \stackrel{\phi_{1}}{\longrightarrow} \mathrm{H}^{0}(\kappa(C), \mathbb{Z} / n) \stackrel{\phi_{2}}{\longrightarrow} \mathrm{H}_{\mathcal{S}}^{1}\left(\mathrm{O}_{C, \mathcal{S}}, \mathbb{Z} / n\right) \stackrel{\phi_{3}}{\longrightarrow} \\
& \stackrel{\phi_{3}}{\longrightarrow} \mathrm{H}^{1}\left(\mathrm{O}_{C, \mathcal{S}}, \mathbb{Z} / n\right) \stackrel{\phi_{4}}{\longrightarrow} \mathrm{H}^{1}(\kappa(C), \mathbb{Z} / n) .
\end{aligned}
$$

We compute $\mathrm{H}^{0}(\kappa(C), \mathbb{Z} / n)=\bigoplus_{i=1}^{m} \mathrm{H}^{0}\left(\kappa\left(C_{i}\right), \mathbb{Z} / n\right)=(\mathbb{Z} / n)^{m}$, and $\mathrm{H}^{0}(C, \mathbb{Z} / n)=$ $(\mathbb{Z} / n)^{N}$ where $N$ is the number of $C$ 's connected components. Since $\mathrm{H}^{0}(C, \mathbb{Z} / n)=$ $\mathrm{H}^{0}(U, \mathbb{Z} / n)$ for all $U$ containing $\mathcal{S}$, and $\mathrm{H}^{q}\left(\mathrm{O}_{C, \mathcal{S}}, \mathbb{Z} / n\right)$ is the direct limit of the $\mathrm{H}^{q}(U, \mathbb{Z} / n)$, we have $\mathrm{H}^{0}(C, \mathbb{Z} / n)=\mathrm{H}^{0}\left(\mathrm{O}_{C, \mathcal{S}}, \mathbb{Z} / n\right)=(\mathbb{Z} / n)^{N}$ by the exactness of the direct limit functor.

We claim $\mathrm{H}_{\mathcal{S}}^{1}\left(\mathrm{O}_{C, \mathcal{S}}, \mathbb{Z} / n\right)$ is a finite free $\mathbb{Z} / n$-module. For by (4.5), for each $z \in \mathcal{S}$ we have an exact sequence

$$
0 \longrightarrow \mathrm{H}^{0}(\kappa(z), \mathbb{Z} / n) \longrightarrow \mathrm{H}^{0}\left(K_{i, z}, \mathbb{Z} / n\right) \oplus \mathrm{H}^{0}\left(K_{j, z}, \mathbb{Z} / n\right) \longrightarrow \mathrm{H}_{z}^{1}\left(\mathrm{O}_{C, z}^{\mathrm{h}}, \mathbb{Z} / n\right) \rightarrow 0 .
$$

This shows $\mathrm{H}_{z}^{1}\left(\mathrm{O}_{C, z}^{\mathrm{h}}, \mathbb{Z} / n\right) \simeq \mathbb{Z} / n$, and since $\mathrm{H}_{\mathcal{S}}^{1}\left(\mathrm{O}_{C, \mathcal{S}}, \mathbb{Z} / n\right)$ is a finite direct sum of these groups, it is a finite free $\mathbb{Z} / n$-module, of rank $|\mathcal{S}|=E$.

The result [14, 27.1] implies that a free $\mathbb{Z} / n$-submodule of a $\mathbb{Z} / n$-module is a direct summand. Therefore we have a decomposition

$$
\mathrm{H}^{0}(\kappa(C), \mathbb{Z} / n) \simeq \operatorname{im}\left(\phi_{1}\right) \oplus \operatorname{im}\left(\phi_{2}\right)
$$

and since $\mathrm{H}^{0}(\kappa(C), \mathbb{Z} / n)$ is a finite free $\mathbb{Z} / n$-module, $\operatorname{im}\left(\phi_{2}\right)$ is a finite free $\mathbb{Z} / n$ module by the structure theorem for finitely generated abelian groups. Similarly, since $\mathrm{H}_{\mathcal{S}}^{1}\left(\mathrm{O}_{C, \mathcal{S}}, \mathbb{Z} / n\right)$ is a finite free $\mathbb{Z} / n$-module,

$$
\mathrm{H}_{\mathcal{S}}^{1}\left(\mathrm{O}_{C, \mathcal{S}}, \mathbb{Z} / n\right) \simeq \operatorname{im}\left(\phi_{2}\right) \oplus \operatorname{cok}\left(\phi_{2}\right)
$$

and since $\operatorname{im}\left(\phi_{2}\right)$ and $\mathrm{H}_{\mathcal{S}}^{1}\left(\mathrm{O}_{C, \mathcal{S}}, \mathbb{Z} / n\right)$ are finite free $\mathbb{Z} / n$-modules, so is $\operatorname{cok}\left(\phi_{2}\right)$. Since $\mathrm{H}^{1}\left(\mathrm{O}_{C, \mathcal{S}}, \mathbb{Z} / n\right)$ is a $\mathbb{Z} / n$-module, it follows that $\operatorname{cok}\left(\phi_{2}\right)$ is a direct summand of $\mathrm{H}^{1}\left(\mathrm{O}_{C, \mathcal{S}}, \mathbb{Z} / n\right)$, again by [14, 27.1]. Thus we have a decomposition

$$
\mathrm{H}^{1}\left(\mathrm{O}_{C, \mathcal{S}}, \mathbb{Z} / n\right) \simeq \operatorname{cok}\left(\phi_{2}\right) \oplus \operatorname{im}\left(\phi_{4}\right) .
$$

Now we set $\Gamma=\operatorname{im}\left(\phi_{4}\right)$, and compute $\operatorname{rk}\left(\operatorname{cok}\left(\phi_{2}\right)\right)=N+|\mathcal{S}|-m=N+E-V=\beta_{C}$. This proves the $q=1$ part of (b).

The $q=0$ case of (b) is in the proof of Lemma 4.2. Suppose $q=2$. To show $\mathrm{H}^{2}\left(\mathrm{O}_{C, \mathcal{S}}, \mu_{n}\right) \rightarrow \mathrm{H}^{2}\left(\kappa(C), \mu_{n}\right)$ is injective, we will show $\mathrm{H}^{1}\left(\kappa(C), \mu_{n}\right) \rightarrow$ $\mathrm{H}_{\mathcal{S}}^{2}\left(\mathrm{O}_{C, \mathcal{S}}, \mu_{n}\right)$ is onto and apply the exactness of (4.3).

For each closed point $z \in C_{1} \cap C_{2} \subset \mathcal{S}$, we have a diagram

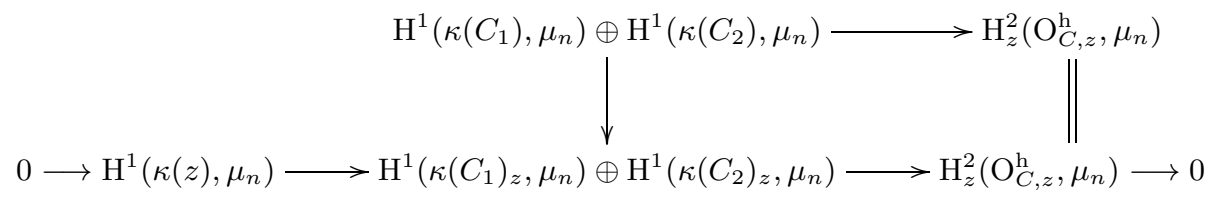

We will show that $\mathrm{H}^{1}\left(\kappa\left(C_{1}\right), \mu_{n}\right) \oplus \mathrm{H}^{1}\left(\kappa\left(C_{2}\right), \mu_{n}\right) \rightarrow \mathrm{H}_{z}^{2}\left(\mathrm{O}_{C, z}^{\mathrm{h}}, \mu_{n}\right)$ is onto, by showing the downarrow is onto. Since $z$ is a regular point of each $C_{i}$, each $\mathrm{O}_{C_{i}, z}$ is a discrete valuation ring with residue field $\kappa(z)$ and fraction field $\kappa\left(C_{i}\right)$, and we have 
a diagram of split short exact sequences

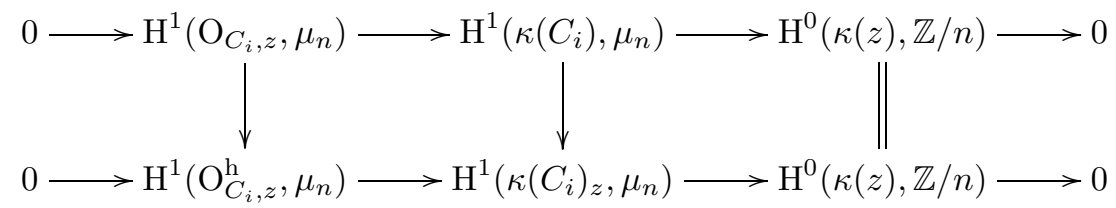

To show the middle downarrow is onto it suffices (by a standard diagram chase) to prove that the left downarrow is onto. Since $\mathrm{O}_{C_{i}, z}^{\mathrm{h}}$ is henselian $\mathrm{H}^{1}\left(\mathrm{O}_{C_{i}, z}^{\mathrm{h}}, \mu_{n}\right)=$ $\mathrm{H}^{1}\left(\kappa(z), \mu_{n}\right)$, and by Kummer theory and Hilbert 90 we have $\mathrm{H}^{1}\left(\mathrm{O}_{C_{i}, z}, \mu_{n}\right)=$ $\mathrm{O}_{C_{i}, z}^{*} / n$ and $\mathrm{H}^{1}\left(\kappa(z), \mu_{n}\right)=\kappa(z)^{*} / n$. Since $\mathrm{O}_{C_{i}, z} \rightarrow \kappa(z)$ is onto and $\mathrm{O}_{C_{i}, z}$ is local, the induced map $\mathrm{O}_{C_{i}, z}^{*} \rightarrow \kappa(z)^{*}$ is onto, hence $\mathrm{H}^{1}\left(\mathrm{O}_{C_{i}, z}, \mu_{n}\right)$ maps onto $\mathrm{H}^{1}\left(\kappa(z), \mu_{n}\right)$. We conclude $\mathrm{H}^{1}\left(\kappa\left(C_{i}\right), \mu_{n}\right) \rightarrow \mathrm{H}^{1}\left(\kappa\left(C_{i}\right)_{z}, \mu_{n}\right)$ is onto. Now each map $\mathrm{H}^{1}\left(\kappa\left(C_{1}\right), \mu_{n}\right) \oplus \mathrm{H}^{1}\left(\kappa\left(C_{2}\right), \mu_{n}\right) \rightarrow \mathrm{H}_{z}^{2}\left(\mathrm{O}_{C, z}^{\mathrm{h}}, \mu_{n}\right)$ is onto.

Suppose $\left(b_{z}\right)_{z \in \mathcal{S}} \in \mathrm{H}_{\mathcal{S}}^{2}\left(\mathrm{O}_{C, \mathcal{S}}, \mu_{n}\right)=\bigoplus_{z \in \mathcal{S}} \mathrm{H}_{z}^{2}\left(\mathrm{O}_{C, z}^{\mathrm{h}}, \mu_{n}\right)$. We have just seen that for each closed point $z \in C_{i} \cap C_{j}$ there exists a pair $\left(\left(a_{i, z}\right),\left(a_{j, z}\right)\right) \in \kappa\left(C_{i}\right)^{*} / n \oplus$ $\kappa\left(C_{j}\right)^{*} / n$ mapping to $b_{z}$, for elements $a_{k, z} \in \mathrm{O}_{C_{k}, z}$, for $k=i, j$. Let $v_{k, z}$ be the discrete valuation on $\kappa\left(C_{k}\right)$ determined by $z$. By standard approximation (e.g. [29, XII.1.2]) there exist elements $a_{k} \in \kappa\left(C_{k}\right)$ such that $v_{k, z}\left(a_{k}-a_{k, z}\right)>v_{k, z}\left(a_{k, z}\right)$ for all $z$. The image of $a_{k}$ in $\kappa\left(C_{k}\right)_{z}^{*} / n$ is then $\left(a_{k, z}\right)$, since the group $U_{z}^{(1)}=\{u \in$ $\left.\kappa\left(C_{k}\right)_{z}^{*}: v_{k, z}(u-1)>0\right\}$ is an $n$-divisible subgroup of the unit group of the henselian field $\kappa\left(C_{k}\right)_{z}$. Therefore the $m$-tuple $\left(\left(a_{k}\right)\right)_{k} \in \bigoplus_{k=1}^{m} \mathrm{H}^{1}\left(\kappa\left(C_{i}\right), \mu_{n}\right)=\mathrm{H}^{1}\left(\kappa(C), \mu_{n}\right)$ maps to $\left(b_{z}\right)$. This proves the induced map $\mathrm{H}^{1}\left(\kappa(C), \mu_{n}\right) \rightarrow \mathrm{H}_{\mathcal{S}}^{2}\left(\mathrm{O}_{C, \mathcal{S}}, \mu_{n}\right)$ is onto, and completes the proof.

We will soon need the following technical lemma in order to replace $X_{0}$ with $C$.

Lemma 4.8. Suppose $A$ is a noetherian ring. Then the natural map (Frac $A)_{\mathrm{red}} \rightarrow$ Frac $\left(A_{\text {red }}\right)$ is an isomorphism if and only if $A$ has no embedded primes.

Proof. Let $f: A \rightarrow A_{\text {red }}=A / N_{A}$ be the natural map, where $N_{A}$ is the nilradical of $A$, let $S=A-\bigcup_{\text {Ass } A} \mathfrak{p}$, where Ass $A$ is the set of associated primes of $A$, and let $T=A-\bigcup_{\operatorname{Min} A} \mathfrak{p}$, where $\operatorname{Min} A$ is the set of minimal primes of $A$. Then $S$ is the set of nonzero-divisors of $A, S \subset T$, and since $\operatorname{Min}\left(A_{\text {red }}\right)=\operatorname{Ass}\left(A_{\text {red }}\right), f(T)$ is the set of nonzero-divisors of $A_{\text {red }}$ (see e.g. [4, Chapter 4]).

We have $(\text { Frac } A)_{\text {red }}=\left(S^{-1} A\right)_{\text {red }}=S^{-1} A / N_{S^{-1} A}$ by definition, and the latter is $S^{-1} A / S^{-1} N_{A}=f(S)^{-1} A_{\text {red }}$ by [4, 3.12]. Since Frac $\left(A_{\text {red }}\right)=f(T)^{-1} A_{\text {red }}$ and $f(S) \subset f(T)$, we have a natural map

$$
(\text { Frac } A)_{\text {red }}=f(S)^{-1} A_{\text {red }} \longrightarrow f(T)^{-1} A_{\text {red }}=\text { Frac }\left(A_{\text {red }}\right) .
$$

By [4. Exercise 3.8(v)] this is an isomorphism if and only if every prime ideal of $A_{\text {red }}$ that meets $f(T)$ also meets $f(S)$. Since every prime of $A$ is the preimage of its image under $f$, the latter condition is equivalent to: Every prime of $A$ that meets $T$ also meets $S$. Every prime that meets $T$ also meets $S$ if and only if $\operatorname{Ass} A \subset \bigcup_{\text {Min } A} \mathfrak{p}$, if and only if $\operatorname{Ass} A \subset \operatorname{Min} A$, by prime avoidance (4, 1.11]). Since always Ass $A \supset$ $\operatorname{Min} A$, we conclude that we have an isomorphism $(\text { Frac } A)_{\text {red }} \stackrel{\sim}{\longrightarrow}$ Frac $\left(A_{\text {red }}\right)$ if and only if $\operatorname{Min} A=\operatorname{Ass} A$, i.e., $A$ has no embedded primes. 
Theorem 4.9. Assume the setup of 2.4. Then for $q \geq 0$ there is a map $\lambda$ : $\mathrm{H}^{q}\left(\mathrm{O}_{C, \mathcal{S}}, \Lambda\right) \rightarrow \mathrm{H}^{q}(F, \Lambda)$ and a commutative diagram

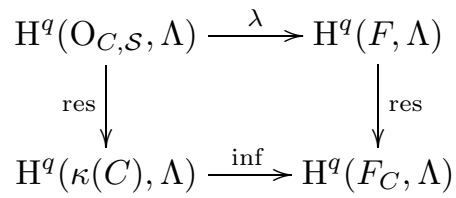

such that if $\alpha_{C} \in \mathrm{H}^{q}\left(\mathrm{O}_{C, \mathcal{S}}, \Lambda\right)$ and $\alpha=\lambda\left(\alpha_{C}\right)$, then:

a) $\alpha$ is defined at the generic point of each $C_{i}$, and $\alpha\left(C_{i}\right)=\alpha_{\kappa\left(C_{i}\right)}=\left(\alpha_{C}\right)_{\kappa\left(C_{i}\right)}$.

b) The ramification locus of $\alpha$ (on $X$ ) is contained in $\mathscr{D}_{\mathcal{S}}$.

c) If $D \in \mathscr{D}_{\mathcal{S}}$ is prime and $z=D \cap C$, then $\partial_{D} \cdot \lambda=\inf _{\kappa(z) \mid \kappa(D)} \cdot \partial_{z}$.

d) If $\alpha_{C}$ is unramified at a closed point $z$, and $D$ is any (horizontal) prime lying over $z$, then $\alpha$ is unramified at $D$, and has value $\alpha(D)=\inf _{\kappa(z) \mid \kappa(D)}\left(\alpha_{C}(z)\right)$.

Proof. Let $D_{C}$ be an effective divisor on $C$ that avoids $\mathcal{S}$, let $D \in \mathscr{D}_{\mathcal{S}}$ be the distinguished lift of $D_{C}$, set $U=X-D$, and set $U_{C}=C-D_{C}$. We will say such $U$ are distinguished. Since $X$ and $D$ are regular and $D$ has pure codimension one, we have $\mathrm{H}^{0}(X) \simeq \mathrm{H}^{0}(U)$, and an exact Gysin sequence

$$
0 \longrightarrow \mathrm{H}^{1}(X) \longrightarrow \mathrm{H}^{1}(U) \stackrel{\partial_{D}}{\longrightarrow} \mathrm{H}^{0}(D,-1) \longrightarrow \mathrm{H}^{2}(X) \longrightarrow \cdots
$$

by Gabber's absolute purity theorem ([15. Theorem 2.1.1]) and the standard construction of the Gysin sequence ([12, Section 3.2]). (Note that the result in [15] is stated for the $\Lambda=\mathbb{Z} / n$ case only, but the result holds in general since the sheaves $\mathscr{H}_{D}^{q}(X)$ and $\mathscr{H}_{D}^{q}(X, \mathbb{Z} / n)$ are locally isomorphic, and the morphism $i^{*} \Lambda(-1) \rightarrow$ $\mathscr{H}_{D}^{2}(X)$ is canonical, where $i: D \rightarrow X$ stands for the closed immersion.) We use the notation $\partial_{D}$ since this map is compatible with the one defined above on $\mathrm{H}^{q}(F)$ when $D$ is prime.

We may replace $X_{0}$ by $C=X_{0, \text { red }}$ in the cohomological computations below since $\Lambda$ is finite and $n$ is prime-to- $p$, by [33, V.2.4(c)] (see also [33, II.3.11]). To substitute $\mathrm{O}_{C, \mathcal{S}}$ and $\kappa(C)$ for $\mathrm{O}_{X_{0}, \mathcal{S}}$ and $\kappa\left(X_{0}\right)$ we must check that the former are the canonical reduced quotients of the latter. But the ring $\mathrm{O}_{X_{0}, \mathcal{S}}$ can be obtained by localizing some affine open subset Spec $A_{0}$ containing $\mathcal{S}$ (which exists since $X_{0} / k$ is projective) with respect to the multiplicative set $T=A_{0}-\bigcup_{z \in \mathcal{S}} \mathfrak{m}_{z}$. Since $\mathrm{O}_{C, \mathcal{S}}$ is obtained by localizing $A_{0, \text { red }}$ with respect to the image of $T$ in $A_{0 \text {,red }}$, we have $\mathrm{O}_{C, \mathcal{S}}=\left(\mathrm{O}_{X_{0}, \mathcal{S}}\right)_{\text {red }}$ since the formation of the nilradical commutes with localization (see e.g. [4, 3.12]).

To show $\kappa(C)=\kappa\left(X_{0}\right)_{\text {red }}$ it suffices to show $X_{0}$ has no embedded points by Lemma 4.8. But if $z$ is any closed point of $X$, then $\mathrm{O}_{X, z}$ is a regular local ring, and a local equation for the closed fiber $\operatorname{Spec}\left(\mathrm{O}_{X, z} \otimes_{R} k\right)$ passing through $z$ is given by the uniformizer $p$ in $R$. Since $\mathrm{O}_{X, z}$ is factorial and at most two components of $X_{0}$ pass through $z$ we have $p=u \pi_{1}^{e_{1}} \pi_{2}^{e_{2}}$ for $u \in \mathrm{O}_{X, z}^{*}$, primes $\pi_{i}$, and numbers $e_{i} \geq 0$. The associated primes of $\mathrm{O}_{X, z} /\left(u \pi_{1}^{e_{1}} \pi_{2}^{e_{2}}\right)$ are evidently just the $\left(\pi_{i}\right)$, which shows $X_{0}$ has no embedded point at $z$.

Since $D_{C}$ is a disjoint union of regular closed points, by (4.7) and the work that immediately precedes it we have $\mathrm{H}^{0}(C) \simeq \mathrm{H}^{0}\left(U_{C}\right)$ and an exact sequence

$$
0 \longrightarrow \mathrm{H}^{1}(C) \longrightarrow \mathrm{H}^{1}\left(U_{C}\right) \stackrel{\partial_{D_{C}}}{\longrightarrow} \mathrm{H}^{0}\left(\kappa\left(D_{C}\right),-1\right) \longrightarrow \mathrm{H}^{2}(C) \longrightarrow \cdots
$$


Thus we have a commutative ladder

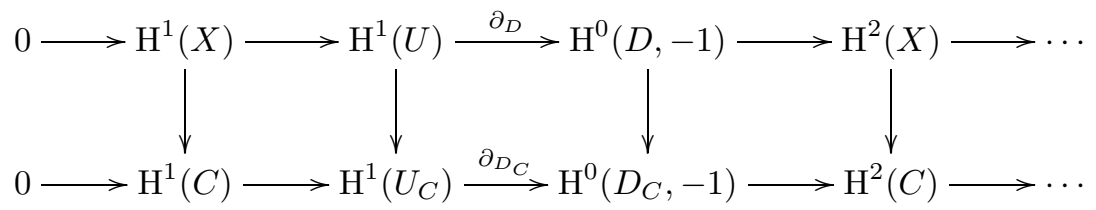

Since $R$ is complete, $\mathrm{H}^{q}(X) \rightarrow \mathrm{H}^{q}(C)$ and $\mathrm{H}^{q}(D,-1) \rightarrow \mathrm{H}^{q}\left(D_{C},-1\right)$ are isomorphisms for $q \geq 0$ by proper base change ([33. VI.Corollary 2.7]). Therefore, in light of the isomorphisms in degree zero and the 5-lemma in degree $q \geq 1$, we obtain isomorphisms

$$
\mathrm{H}^{q}(U) \stackrel{\sim}{\longrightarrow} \mathrm{H}^{q}\left(U_{C}\right)
$$

for $q \geq 0$. Taking the limit over all $U_{C}=C-D_{C}$ (containing $\mathcal{S}$ ) and corresponding $U=X-D$ for $D \in \mathscr{D}_{\mathcal{S}}$ lying over $D_{C}$ yields an isomorphism

$$
\lim _{\longrightarrow} \mathrm{H}^{q}(U) \stackrel{\sim}{\longrightarrow} \underset{\lim }{\longrightarrow} \mathrm{H}^{q}\left(U_{C}\right)=\mathrm{H}^{q}\left(\mathrm{O}_{C, \mathcal{S}}\right) .
$$

On the other hand, for each distinguished $U$ we have a map $\mathrm{H}^{q}(U) \rightarrow \mathrm{H}^{q}(F)$, hence a map $\underset{\lim }{\longrightarrow} \mathrm{H}^{q}(U) \rightarrow \mathrm{H}^{q}(F)$. Composing with the inverse of (4.11) yields our lift

$$
\lambda: \mathrm{H}^{q}\left(\mathrm{O}_{C, \mathcal{S}}\right) \longrightarrow \mathrm{H}^{q}(F) .
$$

Applying cohomology to the commutative diagram

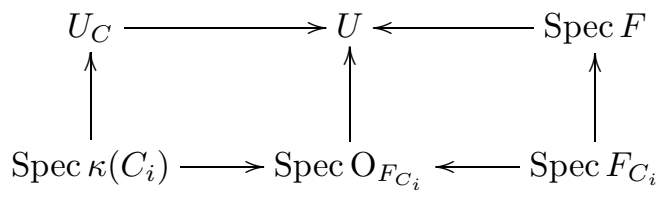

yields a commutative diagram

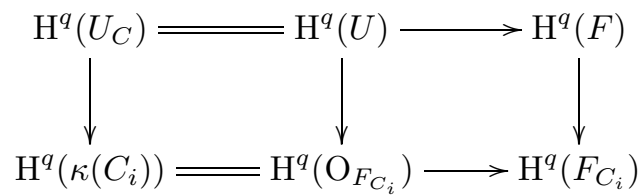

Taking the limit again over $U_{C}$ and $U$ yields the diagram 4.10). If $\alpha_{C} \in \mathrm{H}^{q}\left(\mathrm{O}_{C, \mathcal{S}}\right)$ and $\alpha=\lambda\left(\alpha_{C}\right)$, then since each $U$ contains $\mathcal{S}$ and the generic points of the $C_{i}$, $\alpha$ is defined at these points, and the formula $\alpha\left(C_{i}\right)=\operatorname{res}_{\mathrm{O}_{C, \mathcal{S}} \mid \kappa\left(C_{i}\right)}\left(\alpha_{C}\right)$ follows immediately from (4.10). Since the restriction map $\mathrm{H}^{q}\left(\mathrm{O}_{X, \kappa\left(C_{i}\right)}\right) \rightarrow \mathrm{H}^{q}\left(\kappa\left(C_{i}\right)\right)$ factors through $\mathrm{H}^{q}\left(\mathrm{O}_{F_{C_{i}}}\right)$, we have $\alpha\left(C_{i}\right)=\alpha_{\kappa\left(C_{i}\right)}$. This proves (a).

If $D$ is a horizontal prime divisor not in $\mathscr{D}_{\mathcal{S}}$, then the generic point $\operatorname{Spec} \kappa(D)$ is contained in each distinguished $U$, hence $\lim _{\mathrm{H}} \mathrm{H}^{q}(U) \rightarrow \mathrm{H}^{q}\left(F_{D}\right)$ factors through $\mathrm{H}^{q}\left(\mathrm{O}_{F_{D}}\right)$, which shows $\partial_{D} \cdot \lambda=0$. Thus the ramification locus of any element in the image of $\lambda$ must be contained in $\mathscr{D}_{\mathcal{S}}$, proving (b). Now if $D \in \mathscr{D}_{\mathcal{S}}$ is prime and $z=D \cap C$, then $D$ is the prime spectrum of a complete local ring with residue field $\kappa(z)$, and the isomorphism

$$
\mathrm{H}^{q-1}(D,-1) \stackrel{\sim}{\longrightarrow} \mathrm{H}^{q-1}(z,-1)=\mathrm{H}^{q-1}(\kappa(z),-1)
$$

is the standard identification. Thus the formula $\partial_{D} \cdot \lambda=\inf _{\kappa(z) \mid \kappa(D)} \cdot \partial_{z}$ is immediate by the commutative ladder of Gysin sequences above and the compatibility of $\partial_{D}$ with the residue map on $\mathrm{H}^{q}(F)$, proving (c). 
Suppose $\alpha=\lambda\left(\alpha_{C}\right)$ has ramification locus $D_{\alpha}$; then $D_{\alpha} \in \mathscr{D}_{\mathcal{S}}$. Set $U=X-D_{\alpha}$ and $U_{C}=U \cap C=C-D_{\alpha_{C}}$. If $\alpha_{C}$ is unramified at a point $z$, i.e., $z \in U_{C}$, then $\alpha$ is unramified at every prime divisor $D$ lying over $z$. For if $D \in \mathscr{D}_{\mathcal{S}}$, then $\partial_{D}(\alpha)=\inf \left(\partial_{z}\left(\alpha_{C}\right)\right)$ by the formula just proved, and if $D \notin \mathscr{D}_{\mathcal{S}}$, then $\partial_{D}(\alpha)=0$ since $D_{\alpha} \in \mathscr{D}_{\mathcal{S}}$. Thus if $\alpha_{C}$ is unramified at $z$, and $D$ is a prime divisor lying over $z$, then $U$ contains $z$ and $\operatorname{Spec} \kappa(D)$, hence $U \supset D$. The maps $z=\operatorname{Spec} \kappa(z) \rightarrow U_{C}$ and $D \rightarrow U$ then induce a commutative diagram

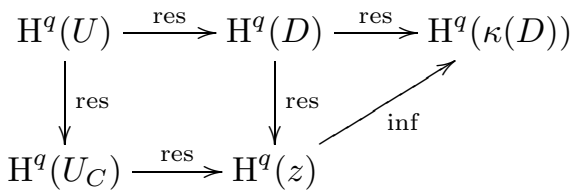

Both vertical downarrows are isomorphisms by the commutative ladder. The inverse of the left one is $\lambda$ by definition, and the composition of the inverse of the right one and the restriction $\mathrm{H}^{q}(D) \rightarrow \mathrm{H}^{q}(\kappa(D))$ is inflation, as shown. The top composition of horizontal restrictions factors through the restriction $\mathrm{H}^{q}(U) \rightarrow \mathrm{H}^{q}\left(\widehat{\mathrm{O}}_{X, D}\right)$ and the bottom factors through the restriction $\mathrm{H}^{q}\left(U_{C}\right) \rightarrow \mathrm{H}^{q}\left(\widehat{\mathrm{O}}_{C, z}\right)$. Since these are restriction maps, the images of $\alpha$ and $\alpha_{C}$ are the values $\alpha(D)$ and $\alpha_{C}(z)$. We conclude $\inf _{\kappa(z) \mid \kappa(D)}\left(\alpha_{C}(z)\right)=\alpha(D)$, as in $(\mathrm{d})$.

Lemma 4.12. Assume the setup of 2.4, let $D \in \mathscr{D}_{\mathcal{S}}$, and suppose $\rho: Y \rightarrow(X, D)$ is a tamely ramified cover. Then there is a map $\lambda_{Y}: \mathrm{H}^{q}\left(\mathrm{O}_{C_{Y}, \mathcal{S}_{Y}}, \Lambda\right) \rightarrow \mathrm{H}^{q}(K(Y), \Lambda)$ inducing a commutative diagram

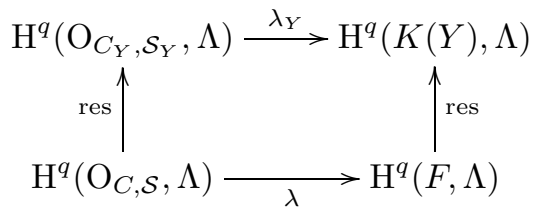

Proof. Since $\rho: Y \rightarrow(X, D)$ is a tamely ramified cover, $Y / R$ is a regular relative curve, each irreducible component of $C_{Y}=\left(Y_{0}\right)_{\text {red }}$ is regular, and exactly two irreducible components of $C_{Y}$ meet at each singular point $z \in \mathcal{S}_{Y}$, all by Lemma 3.1. Thus $Y / R$ satisfies the hypotheses of 2.4, and we have the map $\lambda_{Y}$ by Theorem 4.9. which is defined relative to the set of distinguished divisors $\mathscr{D}_{Y}$ as in Lemma 3.1(c).

Let $U_{C} \subset C$ be any open set containing $\mathcal{S}$, set $D_{C}^{\prime}=C-U_{C}$, and let $D^{\prime} \in \mathscr{D}_{\mathcal{S}}$ be the distinguished divisor lying over $D_{C}^{\prime}$. Then let $U=X-D^{\prime}, V=Y \times_{X} U$, and $V_{C}=U_{C} \times_{U} V$. Then we have a commutative diagram

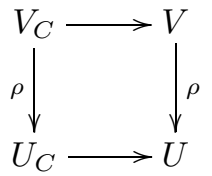


Since $\mathcal{S}_{Y}=\rho^{-1} \mathcal{S}, V$ and $V_{C}$ contain $\mathcal{S}_{Y}$, and applying cohomology yields a commutative diagram

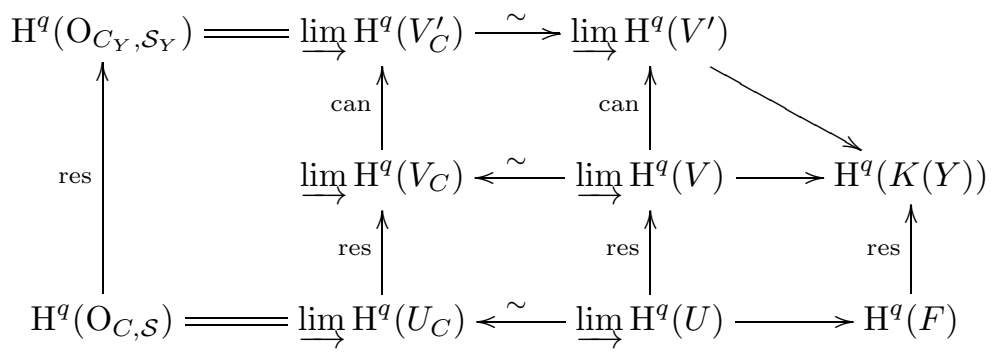

where $V^{\prime}$ runs over all open subsets of $Y$ containing $\mathcal{S}_{Y}$, and $V_{C}^{\prime}=V^{\prime} \times{ }_{X} C$. This yields the diagram of the lemma.

4.13. Since $X$ is noetherian each $f \in F^{*}$ defines a divisor $\operatorname{div} f=\sum v_{D}(f) D$, where the (finite) sum is over prime divisors on $X$. By weak approximation [40, Lemma] there exists a $\pi \in F$ such that

$$
\operatorname{div} \pi=C+H
$$

where $H$ is horizontal and avoids any finite (preassigned) set of points $\mathcal{F}$. We fix such a $\pi$ for $\mathcal{F}$ containing $\mathcal{S}$. Since $v_{C_{i}}(\pi)=1$ for each $i$, the choice of $\pi$ determines a noncanonical Witt decomposition

$$
\mathrm{H}^{q}(\kappa(C), \Lambda) \oplus \mathrm{H}^{q-1}(\kappa(C), \Lambda(-1)) \stackrel{\sim}{\longrightarrow} \mathrm{H}^{q}\left(F_{C}, \Lambda\right)
$$

taking $\left(\alpha_{C}, \theta_{C}\right)$ to $\alpha_{C}+(\pi) \cdot \theta_{C}$, where $\alpha_{C}$ and $\theta_{C}$ are inflated from $\kappa(C)$ to $F_{C}$, $(\pi)$ is the image of $\pi$ in $\mathrm{H}^{1}\left(F_{C}, \mu_{n}\right)$, and $(\pi) \cdot \theta$ is the cup product. Composing this map with the natural maps $\mathrm{H}^{q}\left(\mathrm{O}_{C, \mathcal{S}}, \Lambda\right) \rightarrow \mathrm{H}^{q}(\kappa(C), \Lambda)$ and $\mathrm{H}^{q-1}\left(\mathrm{O}_{C, \mathcal{S}}, \Lambda(-1)\right) \rightarrow$ $\mathrm{H}^{q-1}(\kappa(C), \Lambda(-1))$ yields a homomorphism

$$
\eta_{\pi}: \mathrm{H}^{q}\left(\mathrm{O}_{C, \mathcal{S}}, \Lambda\right) \oplus \mathrm{H}^{q-1}\left(\mathrm{O}_{C, \mathcal{S}}, \Lambda(-1)\right) \longrightarrow \mathrm{H}^{q}\left(F_{C}, \Lambda\right)
$$

defined by $\eta_{\pi}\left(\alpha_{C}, \theta_{C}\right)=\alpha_{C}+(\pi) \cdot \theta_{C}$.

Corollary 4.14. The choice of $\mathscr{D}_{\mathcal{S}}$ and $\pi$ determines for $q \geq 1$ a homomorphism

$$
\lambda: \mathrm{H}^{q}\left(\mathrm{O}_{C, \mathcal{S}}, \Lambda\right) \oplus \mathrm{H}^{q-1}\left(\mathrm{O}_{C, \mathcal{S}}, \Lambda(-1)\right) \longrightarrow \mathrm{H}^{q}(F, \Lambda)
$$

by the rule $\lambda\left(\alpha_{C}+(\pi) \cdot \theta_{C}\right)=\lambda\left(\alpha_{C}\right)+(\pi) \cdot \lambda\left(\theta_{C}\right)$, resulting in a commutative diagram

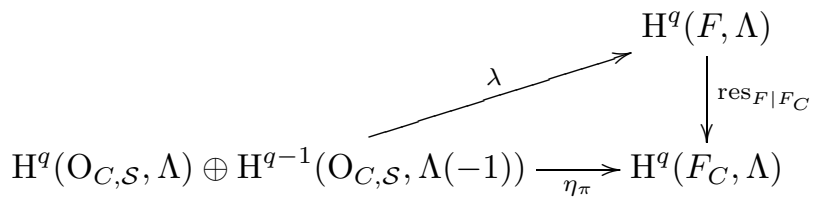


If $D \in \mathscr{D}_{\mathcal{S}}$ and $\rho: Y \rightarrow(X, D)$ is a tamely ramified cover, then we have a commutative diagram

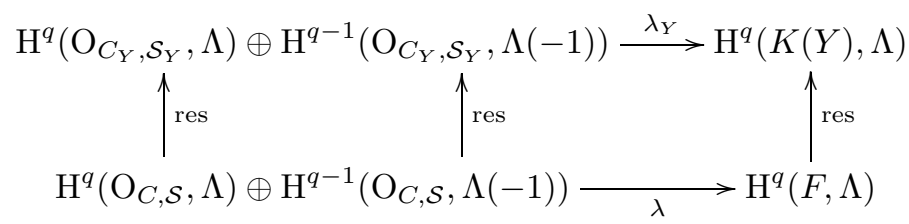

where $\lambda_{Y}$ is defined using $\mathscr{D}_{\mathcal{S}_{Y}}$, $\pi$, and Lemma 4.12.

Proof. This is an immediate consequence of Theorem 4.9 and Lemma 4.12 ,

Remark 4.15. If $X / R$ is smooth, then $\mathcal{S}$ is empty, and $\mathrm{O}_{C, \mathcal{S}}=\kappa(C)$. The map $\eta_{\pi}$ is then an isomorphism, and incorporating it into $\lambda$ we obtain a map

$$
\lambda: \mathrm{H}^{q}\left(F_{C}, \Lambda\right) \longrightarrow \mathrm{H}^{q}(F, \Lambda)
$$

that splits the restriction map. This is the map of [10]. Note however that Corollary 4.14 does not imply a well-defined map $\operatorname{im}\left(\eta_{\pi}\right) \rightarrow \mathrm{H}^{q}(F, \Lambda)$. In fact there is an obstruction, at least part of which lies in the prospect of nontrivial elements of $\operatorname{im}(\lambda) \leq \mathrm{H}^{q}(F, \Lambda)$ that are trivial at $\kappa(C)$. We discuss the $q=1$ case in 4.16 .

4.16. Completely split elements. In [36, 2.1] Saito defines a completely split covering of a noetherian scheme $X$ to be a finite étale cover $Y \rightarrow X$ such that $Y \times_{X} \operatorname{Spec} \kappa(x)=\coprod \operatorname{Spec} \kappa(x)$, for all closed points $x \in X$. We abuse Saito's terminology (see Remark4.18 below) and in the setup of 2.4 denote by $\mathrm{H}_{\mathrm{cs}}^{1}(C, \Lambda)$ the kernel of the map $\mathrm{H}^{1}\left(\mathrm{O}_{C, \mathcal{S}}, \Lambda\right) \rightarrow \mathrm{H}^{1}(\kappa(C), \Lambda)$, so that we have an exact sequence

$$
0 \longrightarrow \mathrm{H}_{\mathrm{cs}}^{1}(C, \Lambda) \longrightarrow \mathrm{H}^{1}\left(\mathrm{O}_{C, \mathcal{S}}, \Lambda\right) \longrightarrow \mathrm{H}^{1}(\kappa(C), \Lambda) \text {. }
$$

Then $\mathrm{H}_{\mathrm{cs}}^{1}(C, \Lambda) \leq \mathrm{H}^{1}(C, \Lambda)$ : For if $\beta \in \mathrm{H}_{\mathrm{cs}}^{1}(C, \Lambda)$, then $\partial_{z}(\beta)=0$ for all closed points $z \in C-\mathcal{S}$ since $\partial_{z}$ factors through $\kappa\left(C_{i}\right)_{z}$. Therefore $\beta$ is defined on $C$, and since $\mathrm{H}^{1}(C, \Lambda) \leq \mathrm{H}^{1}\left(\mathrm{O}_{C, \mathcal{S}}, \Lambda\right)$ by Lemma 4.6(a), $\mathrm{H}_{\mathrm{cs}}^{1}(C, \Lambda) \leq \mathrm{H}^{1}(C, \Lambda)$, as claimed. Let $\mathrm{H}_{\mathrm{cs}}^{1}(X, \Lambda)$ denote the preimage of $\mathrm{H}_{\mathrm{cs}}^{1}(C, \Lambda)$ under the proper base change isomorphism $\mathrm{H}^{1}(X, \Lambda) \stackrel{\sim}{\longrightarrow} \mathrm{H}^{1}(C, \Lambda)$.

Proposition 4.17. Assume the setup of 2.4. Then elements of $\mathrm{H}_{\mathrm{cs}}^{1}(C, \Lambda)$ are trivial at all points of $C$, and the nontrivial elements of $\mathrm{H}_{\mathrm{cs}}^{1}(X, \Lambda)$ are trivial at all points of $X$ except for the generic point $\operatorname{Spec} F$, where they are nontrivial.

Proof. Suppose $\beta_{C} \in \mathrm{H}_{\mathrm{cs}}^{1}(C)$. Then $\beta_{C}$ is trivial at each generic point of $C$ by definition of $\mathrm{H}_{\mathrm{cs}}^{1}(C)$. If $z \in C$ is a closed point lying on the irreducible component $C_{i}$, then the map $\mathrm{H}^{1}(C) \rightarrow \mathrm{H}^{1}(\kappa(z))$ factors through $\mathrm{H}^{1}\left(C_{i}\right)$. Since $C_{i}$ is regular the map $\mathrm{H}^{1}\left(C_{i}\right) \rightarrow \mathrm{H}^{1}\left(\kappa\left(C_{i}\right)\right)$ is injective by purity, and consequently $\beta_{C}(z)=0$ by definition. Thus the elements of $\mathrm{H}_{\mathrm{cs}}^{1}(C)$ are trivial at all points of $C$.

Suppose $\beta=\lambda\left(\beta_{C}\right) \in \mathrm{H}_{\mathrm{cs}}^{1}(X)$. If $x \in X$ is a generic point of some irreducible component $C_{i}$ of $C$, then the image of $\beta$ in $\mathrm{H}^{1}\left(\kappa\left(C_{i}\right)\right)$ is zero since the map $\mathrm{H}_{\mathrm{cs}}^{1}(X) \rightarrow \mathrm{H}^{1}\left(\kappa\left(C_{i}\right)\right)$ factors through $\mathrm{H}_{\mathrm{cs}}^{1}(C)$. If $x$ is the generic point of a horizontal divisor $D$ with closed point $z$, then $\beta(D)=\inf _{\kappa(z) \mid \kappa(D)}\left(\beta_{C}(z)\right)$ by Theorem $4.9(\mathrm{~d})$, and this is zero since $\beta_{C}(z)=0$. If $z$ is a closed point of $X$, then $z$ is on $C$, and the map $\mathrm{H}_{\mathrm{cs}}^{1}(X) \rightarrow \mathrm{H}^{1}(\kappa(z))$ factors through $\mathrm{H}_{\mathrm{cs}}^{1}(C)$, hence $\beta$ is trivial at $z$. Finally, since $X$ is a regular noetherian scheme the map $\mathrm{H}^{1}(X) \rightarrow \mathrm{H}^{1}(F)$ is injective by purity, hence $\beta$ is nontrivial at the generic point of $X$. 
Remark 4.18. Proposition 4.17 shows the elements of $\mathrm{H}_{\mathrm{cs}}^{1}(C, \mathbb{Z} / n)$ are completely split in the sense of [36], since they are split at all closed points. However, $\mathrm{H}_{\mathrm{cs}}^{1}(C, \mathbb{Z} / n)$ does not contain elements that are split at all closed points but nontrivial at generic points of $C$. Such elements do not exist if $k$ is finite, as shown by Saito in [36. Theorem 2.4], since then the $C_{i}$ have no nontrivial completely split covers, essentially by Cebotarev's density theorem (see [35, Lemma 1.7]).

\section{Applications in the Brauer group}

5.1. Cyclic covers. If $U$ is any scheme, and $\bar{u}$ is a geometric point, the fiber functor defines a category equivalence between (finite) étale covers of $U$ and finite continuous $\pi_{1}(U, \bar{u})$-sets, yielding a canonical isomorphism

$$
\mathrm{H}^{1}(U, \mathbb{Z} / n) \simeq \mathrm{H}^{1}\left(\pi_{1}(U, \bar{u}), \mathbb{Z} / n\right)=\operatorname{Hom}_{\text {cont }}\left(\pi_{1}(U, \bar{u}), \mathbb{Z} / n\right)
$$

(see [13, I.2.11]). If $\theta \in \mathrm{H}^{1}(U, \mathbb{Z} / n)$, we will write $U[\theta]$ for the finite cyclic étale cover determined by $\theta$. If $U=\operatorname{Spec} A$ is affine, we will write $A[\theta]$ for the corresponding ring, or $A(\theta)$ if $A$ is a field. If $U$ is a connected normal scheme, and $\theta \in \mathrm{H}^{1}(U, \mathbb{Z} / n)$ has order $m$, then $U[\theta]$ is a disjoint sum of $n / m$ connected $\mathbb{Z} / m$-Galois covers of $U$.

If $V \rightarrow U$ is a morphism and $\bar{v}$ is a geometric point of $V$ (hence of $U$ ), base extension defines a homomorphism $\pi_{1}(V, \bar{v}) \rightarrow \pi_{1}(U, \bar{v})$, inducing the restriction map $\mathrm{H}^{1}(U, \mathbb{Z} / n) \rightarrow \mathrm{H}^{1}(V, \mathbb{Z} / n)$ via (5.2). If $\theta \in \mathrm{H}^{1}(U, \mathbb{Z} / n)$ maps to $\theta_{V} \in \mathrm{H}^{1}(V, \mathbb{Z} / n)$, then $U[\theta] \times_{U} V=V\left[\theta_{V}\right]$.

In the setup of 2.4 if $U \subset X$ is an open subscheme, then since $X$ is excellent and regular, the map $\mathrm{H}^{1}(U, \mathbb{Z} / n) \rightarrow \mathrm{H}^{1}(F, \mathbb{Z} / n)$ is injective (see e.g. the proof of [12, Corollary 3.4.2]). Thus if $\theta \in \mathrm{H}^{1}(F, \mathbb{Z} / n)$ and $\theta$ is defined on $U \subset X$, then we may view $\theta$ as an element of $\mathrm{H}^{1}(U, \mathbb{Z} / n)$. Then $U[\theta] \times_{U} \operatorname{Spec} F=\operatorname{Spec} F(\theta)$, and since $U[\theta] \rightarrow U$ is étale, $F(\theta)$ is the total fraction ring of $U[\theta]$, and $U[\theta]$ is the normalization of $U$ in $\operatorname{Spec} F(\theta)$.

Lemma 5.3. Assume the setup of 2.4. Suppose $\theta_{C} \in \mathrm{H}^{1}\left(\mathrm{O}_{C, \mathcal{S}}, \mathbb{Z} / n\right)$ has ramification divisor $D_{C}$ on $C, \theta=\lambda\left(\theta_{C}\right), D \in \mathscr{D}_{\mathcal{S}}$ is the distinguished lift of $D_{C}$, and $Y$ is the normalization of $X$ in $F(\theta)$. Then $\rho: Y \rightarrow(X, D)$ is a tamely ramified cover whose restriction to $C$ is a tamely ramified cover $\rho_{C}: C_{Y} \rightarrow\left(C, D_{C}\right)$, and $\mathrm{O}_{C_{Y}, \mathcal{S}_{Y}}=\mathrm{O}_{C, \mathcal{S}}\left[\theta_{C}\right]$.

Proof. The lift $\theta$ is tamely ramified with respect to $D$ by Theorem 4.9 If $\theta \in$ $\mathrm{H}^{1}(F, \mathbb{Z} / n), v$ is any valuation on $F$, and $w$ is an extension of $v$ to $F(\theta)$, then the ramification index $e(w / v)$ equals $\left|\partial_{v}(\theta)\right|$, by e.g. [16, Chapter II]. Therefore, since $X$ is regular, $\rho: Y \rightarrow(X, D)$ is a tamely ramified cover by Lemma 3.2

Let $U=X-D, V=U \times_{X} Y, U_{C}=U \times_{X} C \subset C$ and $V_{C}=V \times_{U} U_{C} \subset C_{Y}$. The construction of the map $\lambda$ in (4.11) shows that $\theta_{C}$ and $\theta$ may be viewed as elements of $\mathrm{H}^{1}\left(U_{C}, \mathbb{Z} / n\right)$ and $\mathrm{H}^{1}(U, \mathbb{Z} / n)$, respectively, and $\operatorname{res}_{U \mid U_{C}}(\theta)=\theta_{C}$. Since $Y$ is the normalization of $X$ in $F(\theta), V$ is the normalization of $U$ in $F(\theta)$, hence $V=U[\theta]$. Since $\operatorname{res}_{U \mid U_{C}}: \mathrm{H}^{1}(U, \mathbb{Z} / n) \rightarrow \mathrm{H}^{1}\left(U_{C}, \mathbb{Z} / n\right)$ is induced on covers by base change, we conclude $V_{C}=V \times_{U} U_{C}=U_{C}\left[\theta_{C}\right]$. Now if $U_{C}^{\prime} \subset U_{C}$ is any open set containing $\mathcal{S}$, then we may view $\theta_{C}$ as an element of $\mathrm{H}^{1}\left(U_{C}^{\prime}, \mathbb{Z} / n\right)$, and $V_{C}^{\prime}=V_{C} \times_{U_{C}} U_{C}^{\prime}=U_{C}^{\prime}\left[\theta_{C}\right]$. Taking the limit over all such $U_{C}^{\prime}$ yields the ring $\mathrm{O}_{C, \mathcal{S}}\left[\theta_{C}\right]$, and since $\mathcal{S}_{Y}=\rho^{-1} \mathcal{S}$, this is $\mathrm{O}_{C_{Y}, \mathcal{S}_{Y}}$ by [4, Exercise 3.8] (observe that $\left.\operatorname{Spec} \mathrm{O}_{C_{Y}, \mathcal{S}_{Y}}=\operatorname{Spec} \mathrm{O}_{C, \mathcal{S}} \times_{C} C_{Y}\right)$. 
The map $C_{Y} \rightarrow C$ induced by $Y \rightarrow X$ is finite and étale over $U_{C}=C-D_{C}$ by base change. Each irreducible component of $C_{Y}$ is regular by Lemma 3.1 and dominates an irreducible component of $C$, hence $C_{Y} \rightarrow\left(C, D_{C}\right)$ is a totally ramified cover by the definition in 2.3 . This completes the proof.

5.4. Index. Let $\pi \in F$ and $\eta_{\pi}$ be as in 4.13, with $q=2$. Let $\delta_{C}=\alpha_{C}+(\pi) \cdot \theta_{C}$ be in $\mathrm{H}^{2}\left(F_{C}, \mu_{n}\right)$, and let $\delta_{C_{i}}=\alpha_{C_{i}}+(\pi) \cdot \theta_{C_{i}}$ be the image of $\delta_{C}$ in $\mathrm{H}^{2}\left(F_{C_{i}}, \mu_{n}\right)$. By the (well-known) Nakayama-Witt index formula we have $\operatorname{ind}\left(\delta_{C_{i}}\right)=\left|\theta_{C_{i}}\right|$.

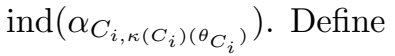

$$
\operatorname{ind}\left(\alpha_{C}, \theta_{C}\right)=\operatorname{lcm}_{i}\left\{\operatorname{ind}\left(\delta_{C_{i}}\right)\right\}
$$

Theorem 5.5. Assume the setup of 2.4 with $R=\mathbb{Z}_{p}$, and let $\lambda$ be the map of Corollary 4.14, Then $\operatorname{ind}\left(\lambda\left(\alpha_{C}, \theta_{C}\right)\right)=\operatorname{ind}\left(\alpha_{C}, \theta_{C}\right)$.

Proof. We adopt the notation of [5.4, and set $\delta=\lambda\left(\alpha_{C}, \theta_{C}\right), \alpha=\lambda\left(\alpha_{C}\right)$, and $\theta=\lambda\left(\theta_{C}\right)$, so that $\delta=\alpha+(\pi) \cdot \theta$. By primary decomposition we may assume $n$ is a power of a prime $\ell$. In Corollary 4.14 we have defined for every $X / \mathbb{Z}_{p}$ satisfying the setup of 2.4 a map $\lambda: \mathrm{H}^{2}\left(\mathrm{O}_{C, \mathcal{S}}, \mu_{n}\right) \oplus \mathrm{H}^{1}\left(\mathrm{O}_{C, \mathcal{S}}, \mathbb{Z} / n\right) \longrightarrow \mathrm{H}^{2}\left(F, \mu_{n}\right)$, which is constructed relative to $\pi$ and $\mathscr{D}_{\mathcal{S}}$.

To show that $\lambda$ preserves index, we proceed by induction on ind $\left(\alpha_{C}, \theta_{C}\right)$. Assume first that $\operatorname{ind}\left(\alpha_{C}, \theta_{C}\right)=1$, i.e., that all $\alpha_{C_{i}}, \theta_{C_{i}}$ are trivial. Then $\theta_{C} \in \mathrm{H}_{\mathrm{cs}}^{1}(C, \mathbb{Z} / n)$ and $\theta \in \mathrm{H}_{\mathrm{cs}}^{1}(X, \mathbb{Z} / n)$ by 4.16 , and $\alpha_{C}=0$ by Lemma 4.6. hence $\alpha=0$. To show that $\delta=(\pi) \cdot \theta$ is trivial we compute ramification:

$$
\partial_{D}((\pi) \cdot \theta)=v_{D}(\pi) \cdot \theta_{F_{D}}-(\pi) \cdot \partial_{D}(\theta)+(-1) \cdot v_{D}(\pi) \cdot \partial_{D}(\theta) .
$$

Since $\theta \in \mathrm{H}_{\mathrm{cs}}^{1}(X, \mathbb{Z} / n), \partial_{D}(\theta)=0$ and $\theta_{F_{D}}=0$ by Proposition 4.17, hence $\partial_{D}((\pi)$. $\theta)=0$, as desired.

Now assume ind $\left(\alpha_{C}, \theta_{C}\right)=N>1$, and that $\lambda$ preserves index on elements of $\ell$-power index less than $N$. Note this hypothesis applies to any $X$ satisfying the setup of 2.4. We construct a tamely ramified cover $\rho: Y \rightarrow X$ of degree $\ell$ as follows. Set $\phi_{C_{i}}=\left(\left|\theta_{C_{i}}\right| / \ell\right) \theta_{C_{i}} \in \mathrm{H}^{1}\left(\kappa\left(C_{i}\right), \mathbb{Z} / \ell\right)$ if $\theta_{C_{i}} \neq 0$, and otherwise use Grunwald-Wang's theorem to produce an element $\phi_{C_{i}} \in \mathrm{H}^{1}\left(\kappa\left(C_{i}\right), \mathbb{Z} / \ell\right)$ of order $\ell$ such that $\phi_{C_{i}, z} \neq 0$ whenever $\partial_{z}\left(\alpha_{C_{i}}\right) \neq 0$, and if $z \in \mathcal{S}$ is on $C_{i} \cap C_{j}, \phi_{C_{i}, z}=\theta_{C_{j}, z}$. Then there exists an element $\phi_{C}=\left(\phi_{C_{i}}\right)_{i} \in \mathrm{H}^{1}\left(\mathrm{O}_{C, \mathcal{S}}, \mathbb{Z} / \ell\right)$ by Lemma 4.2, and we obtain an element $\phi=\lambda\left(\phi_{C}\right) \in \mathrm{H}^{1}(F, \mathbb{Z} / \ell)$. Let $\rho: Y \rightarrow X$ be the normalization of $X$ in $F(\theta)$, a tamely ramified cover by Lemma [5.3. Then $Y / \mathbb{Z}_{p}$ satisfies 2.4 by Lemma 3.1 .

We claim ind $\left(\alpha_{C_{Y}}, \theta_{C_{Y}}\right)=\ell^{-1} \operatorname{ind}\left(\alpha_{C}, \theta_{C}\right)$. By Lemma 5.3 we have $\kappa\left(C_{Y}\right)=$ $\kappa(C)\left(\phi_{C}\right)$, and in particular $\kappa\left(C_{i, Y}\right)=\kappa\left(C_{i}\right)\left(\phi_{C_{i}}\right)$ is a field. Since $\pi$ has multiplicity one at each irreducible component of $C_{Y}$ by Lemma 3.1. we compute

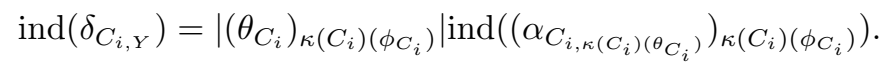

By construction, restriction of each nonzero $\delta_{C_{i}}$ to $\kappa\left(C_{i}\right)\left(\phi_{C_{i}}\right)$ either lowers the order of $\theta_{C_{i}}$ by $\ell$, if $\theta_{C_{i}} \neq 0$, or otherwise, since $\kappa\left(C_{i}\right)$ is a global field, lowers the index of $\alpha_{C_{i, \kappa\left(C_{i}\right)\left(\theta_{C_{i}}\right)}}$ by $\ell$ (by the local-global splitting principle in class field theory). Therefore $\operatorname{ind}\left(\delta_{C_{i, Y}}\right)=\ell^{-1} \operatorname{ind}\left(\delta_{C_{i}}\right)$, hence

$$
\operatorname{ind}\left(\alpha_{C_{Y}}, \theta_{C_{Y}}\right)=\ell^{-1} \operatorname{ind}\left(\alpha_{C}, \theta_{C}\right) \text {. }
$$

This proves the claim. 
Since $Y$ satisfies 2.4 we have $\operatorname{ind}\left(\lambda_{Y}\left(\alpha_{C_{Y}}, \theta_{C_{Y}}\right)\right)=\operatorname{ind}\left(\alpha_{C_{Y}}, \theta_{C_{Y}}\right)$ by the induction hypothesis, and $\lambda_{Y}\left(\alpha_{C_{Y}}, \theta_{C_{Y}}\right)=\lambda\left(\alpha_{C}, \theta_{C}\right)_{K(Y)}$ by the commutative diagram of Lemma 4.12. Therefore $\operatorname{ind}\left(\lambda\left(\alpha_{C}, \theta_{C}\right)_{K(Y)}\right)=\ell^{-1} \operatorname{ind}\left(\alpha_{C}, \theta_{C}\right)$, hence $\operatorname{ind}\left(\lambda\left(\alpha_{C}, \theta_{C}\right)\right) \leq[K(Y): F] \operatorname{ind}\left(\lambda\left(\alpha_{C}, \theta_{C}\right)_{K(Y)}\right)=\ell \cdot \ell^{-1} \operatorname{ind}\left(\alpha_{C}, \theta_{C}\right)=\operatorname{ind}\left(\alpha_{C}, \theta_{C}\right)$.

On the other hand, $\lambda\left(\alpha_{C}, \theta_{C}\right)_{F_{C_{i}}}=\delta_{C_{i}}$ has index $\operatorname{ind}\left(\alpha_{C}, \theta_{C}\right)$ for some $i$ by definition, hence $\operatorname{ind}\left(\lambda\left(\alpha_{C}, \theta_{C}\right)\right) \geq \operatorname{ind}\left(\alpha_{C}, \theta_{C}\right)$. Therefore we have equality, proving the theorem.

Though we do not know how to lift all of $\mathrm{H}^{2}\left(F_{C}, \mu_{n}\right)$ to $\mathrm{H}^{2}\left(F, \mu_{n}\right)$, we now have the following.

Corollary 5.6. Assume the setup of Theorem 5.5. Let $\mathrm{G}_{\pi}^{2} \leq \mathrm{H}^{2}\left(F_{C}, \mu_{n}\right)$ denote the image of $\eta_{\pi}$ in 4.13. Then $\mathrm{G}_{\pi}^{2} \simeq \mathrm{H}^{2}\left(\mathrm{O}_{C, \mathcal{S}}, \mu_{n}\right) \oplus \Gamma$, where $\Gamma \leq \mathrm{H}^{1}\left(\mathrm{O}_{C, \mathcal{S}}, \mathbb{Z} / n\right)$ is as in Lemma 4.6, and the map $\lambda$ of Corollary 4.14 factors through $\mathrm{H}^{2}\left(F_{C}, \mu_{n}\right)$ to induce an injection

$$
\lambda: \mathrm{G}_{\pi}^{2} \longrightarrow \mathrm{H}^{2}\left(F, \mu_{n}\right)
$$

that preserves index and splits the restriction $\operatorname{res}_{F \mid F_{C}}$.

Proof. We have $\operatorname{ker}\left(\eta_{\pi}\right)=\mathrm{H}_{\mathrm{cs}}^{1}(C, \mathbb{Z} / n)$ by Lemma 4.6 (b), hence in the notation of Lemma 4.6 we have an isomorphism $\mathrm{H}^{2}\left(\mathrm{O}_{C, \mathcal{S}}, \mu_{n}\right) \oplus \Gamma \simeq \mathrm{G}_{\pi}^{2}$. Since $\lambda\left(\operatorname{ker}\left(\eta_{\pi}\right)\right)=0$ by the base case of the induction in Theorem [5.5. we have an induced map $\lambda$ : $\mathrm{G}_{\pi}^{2} \longrightarrow \mathrm{H}^{2}\left(F, \mu_{n}\right)$; it preserves index by Theorem [5.5, and splits the restriction map by the commutative diagram in Theorem 4.9

\section{Noncrossed PROdUCtS AND IndeCOMPosable Division ALGEBRAS}

We apply Theorem 5.5 to prove the existence of noncrossed products and indecomposable division algebras over the function field $F$ of any $p$-adic curve. Noncrossed products over $K(t)$ for $K$ a local field were first constructed in [8, and then constructed more systematically over the function field of a smooth relative $\mathbb{Z}_{p}$-curve in 10 . Indecomposable division algebras of unequal period and index were also constructed in [10], over the same types of fields. Modulo gluing, the method we use below is the same as the one used in [10, Theorem 4.3, Corollary 4.8]. We first present some background; for additional discussion, see e.g. [5], 2], or 38 .

Let $\mathrm{Z}^{2}\left(\mathrm{G}_{F}, F_{\text {sep }}^{*}\right)$ denote the group of continuous Galois 2-cocycles. For each $f$ there exists a unique maximal open normal subgroup $U \triangleleft \mathrm{G}_{F}$ such that $f(s U, t U)=$ $f(s, t)$ for all $s, t \in \mathrm{G}_{F}$. If $L / F$ is the finite Galois extension with group $G=\mathrm{G}_{F} / U$, then $f$ is inflated from $\mathrm{Z}^{2}\left(G, L^{*}\right)$, and $f$ defines a central simple $F$-algebra $A_{f}$ of degree $[L: F]$ via the the crossed product construction:

$$
A_{f}=\sum_{s \in G} L u_{s}: u_{s} u_{t}=f(s, t) u_{s t}, \quad u_{s} x=s(x) u_{s}, \quad \forall x \in L .
$$

Here the $u_{s}$ 's are formal basis elements indexed by $G$. Let $\operatorname{CSA}(F)$ be the set of $F$-isomorphism classes of central simple $F$-algebras. The assignment $f \mapsto A_{f}$ defines a set map

$$
\rho: \mathrm{Z}^{2}\left(\mathrm{G}_{F}, F_{\text {sep }}^{*}\right) \longrightarrow \mathrm{CSA}(F) .
$$

It can be shown that $L$ is a maximal commutative (étale) subalgebra of $A_{f}$, and we define a crossed product to be any central simple $F$-algebra with a Galois maximal 
commutative étale subalgebra. The crossed product problem over $F$ is to determine whether every central simple $F$-algebra is a crossed product. In particular the problem is to determine whether every $F$-division algebra is a crossed product, or, in other words, whether there exist noncrossed product $F$-division algebras. It was long believed that all central simple algebras were crossed products, until Amitsur discovered noncrossed products in 1 . The set map $\rho$ induces a surjective group homomorphism $\mathrm{Z}^{2}\left(\mathrm{G}_{F}, F_{\mathrm{sep}}^{*}\right) \rightarrow \operatorname{Br}(F)$ with kernel $\mathrm{B}^{2}\left(\mathrm{G}_{F}, F_{\mathrm{sep}}^{*}\right)$, giving the wellknown isomorphism

$$
\mathrm{H}^{2}\left(\mathrm{G}_{F}, F_{\text {sep }}^{*}\right) \stackrel{\sim}{\longrightarrow} \operatorname{Br}(F) .
$$

It follows by Wedderburn's theorem that for every $F$-division algebra $D$ there exists a number $r$ such that $\mathrm{M}_{r}(D)$ is a crossed product.

We say an $F$-division algebra $D$ is indecomposable if it does not properly contain a nontrivial $F$-subalgebra that is also central over $F$, or equivalently if it is not an $F$-tensor product of two nontrivial $F$-division algebras. It is not hard to show that all division algebras of composite period are decomposable into their primary components, and that all division algebras of equal prime-power period and index are indecomposable. The first indecomposable division algebras of unequal primepower period and index appeared in [3] and in [37. These results showed that decomposability does not fully "account" for the phenomenon of unequal period and index in division algebras.

Theorem 6.1. Let $F / \mathbb{Q}_{p}$ be a finitely generated field extension of transcendence degree one. Let $X / \mathbb{Z}_{p}$ be a regular relative curve with function field $F$, let $C_{1}$ be a reduced irreducible component of the closed fiber, let $\ell \neq p$ be a prime, and let $r$ and $s$ be numbers that are maximal such that $\mu_{\ell^{r}} \subset \kappa\left(C_{1}\right)$ and $\mu_{\ell^{s}} \subset \kappa\left(C_{1}\right)\left(\mu_{\ell^{r+1}}\right)$. Then there exist noncrossed product $F$-division algebras of period and index as low as $\ell^{s+1}$ if $r=0$, and $\ell^{2 r+1}$ if $r \neq 0$.

Proof. We may assume (without changing $r$ and $s$ ) that $C$ has regular irreducible components, at most two of which meet at any closed point of $X$. The idea is to use the (known) existence of such algebras over $F_{C_{1}}$ to produce a class in $\mathrm{G}_{\pi}^{2}$, and then apply Corollary 5.6 to prove existence over $F$.

By [10, Theorem 4.7], if $F$ admits a smooth model $X$, then there exist noncrossed product division algebras over $F_{C}$ of period and index as low as $\ell^{s+1}$ if $r=0$, and $\ell^{2 r+1}$ if $r>0$. The resulting Brauer class has the form $\alpha_{C}+(\pi) \cdot \theta_{C} \in \mathrm{H}^{2}\left(F_{C}, \mu_{n}\right)$, where $\alpha_{C} \in \mathrm{H}^{2}\left(\kappa(C), \mu_{n}\right)$ and $\theta_{C} \in \mathrm{H}^{1}(\kappa(C), \mathbb{Z} / n)$. A look at the construction, which proceeds exactly as in [6. Theorem 1], shows we may pre-assign values at any finite set of points of $C$. Thus we may produce a noncrossed product $F_{C_{1}}$-division algebra $D_{F_{C_{1}}}$ with class $\delta_{C_{1}}=\alpha_{C_{1}}+(\pi) \cdot \theta_{C_{1}}$ of the desired period and index, such that $\alpha_{C_{1}, z}=0$ and $\theta_{C_{1}, z}=0$ at all $z \in \mathcal{S} \cap C_{1}$. Set $\alpha_{C_{i}}=0$ and $\theta_{C_{i}}=0$ for $i>1$. Then $\alpha_{C}=\left(\alpha_{C_{i}}\right)_{i} \in \mathrm{H}^{2}\left(\mathrm{O}_{C, \mathcal{S}}, \mu_{n}\right)$ and $\theta_{C}=\left(\theta_{C_{i}}\right)_{i} \in \mathrm{H}^{1}\left(\mathrm{O}_{C, \mathcal{S}}, \mathbb{Z} / n\right)$ by Lemma 4.2, and we have an element

$$
\delta_{C}=\alpha_{C}+(\pi) \cdot \theta_{C} \in \mathrm{G}_{\pi}^{2} \leq \mathrm{H}^{2}\left(F_{C}, \mu_{n}\right)
$$

whose restriction to $F_{C_{1}}$ is $\delta_{C_{1}}$. Then $\delta_{C}$ lifts to $\delta=\lambda\left(\delta_{C}\right) \in \mathrm{H}^{2}\left(F, \mu_{n}\right)$, and $\operatorname{ind}(\delta)=\operatorname{ind}\left(\delta_{C}\right)=\operatorname{ind}\left(\delta_{C_{1}}\right)$ by Corollary 5.6 and the definition of index over $\kappa(C)$. Let $D$ be the $F$-division algebra associated to $\delta$. Since $\operatorname{res}_{F \mid F_{C_{1}}}(\delta)=\delta_{C_{1}}$ (also by Corollary [5.6) and $\operatorname{ind}(\delta)=\operatorname{ind}\left(\delta_{C_{1}}\right), D \otimes_{F} F_{C_{1}}$ is the (noncrossed product) $F_{C_{1}}$ division algebra $D_{F_{C_{1}}}$ associated to $\delta_{C_{1}}$. If $L / F$ is a Galois maximal subfield of $D$, 
then $L \otimes_{F} F_{C_{1}} / F_{C_{1}}$ is a Galois maximal subfield of $D_{F_{C_{1}}}$ by degree count. Since $D_{F_{C_{1}}}$ is a noncrossed product this would be a contradiction, and we conclude $D$ has no Galois maximal subfields. Therefore $D$ is a noncrossed product $F$-division algebra.

Theorem 6.2. Let $F / \mathbb{Q}_{p}$ be a finitely generated field extension of transcendence degree one, and let $\ell \neq p$ be a prime. Then there exist indecomposable F-division algebras of (period,index $)=\left(\ell^{a}, \ell^{b}\right)$, for any numbers $a$ and $b$ satisfying $1 \leq a \leq b \leq$ $2 a-1$.

Proof. Let $X, C, C_{i}$, and $\mathcal{S}$ be as in 2.4. The construction over $F_{C_{1}}$ proceeds exactly as in [10, Proposition 4.2] and [7, and we obtain an indecomposable $F_{C_{1}}$ division algebra $D_{F_{C_{1}}}$ of (period,index) $=\left(\ell^{a}, \ell^{b}\right)$, for any numbers $a$ and $b$ satisfying $1 \leq a \leq b \leq 2 a-1$. The division algebra $D_{F_{C_{1}}}$ is associated to a class $\delta_{C_{1}}=$ $\alpha_{C_{1}}+(\pi) \cdot \theta_{C_{1}} \in \mathrm{H}^{2}\left(F_{C_{1}}, \mu_{n}\right)$. The construction of [10] allows us to assume the components $\alpha_{C_{1}}$ and $\theta_{C_{1}}$ are zero at the singular points $\mathcal{S} \cap C_{1}$, so that by Lemma 4.2 we obtain a class $\delta_{C}=\alpha_{C}+(\pi) \cdot \theta_{C}$ in $\mathrm{G}_{\pi}^{2} \leq \mathrm{H}^{2}\left(F_{C}, \mu_{n}\right)$ whose first component is $\delta_{C_{1}}=\alpha_{C_{1}}+(\pi) \cdot \theta_{C_{1}}$, and whose other components are zero. This class lifts to a class $\delta=\lambda\left(\delta_{C}\right)$, and $\operatorname{ind}(\delta)=\operatorname{ind}\left(\delta_{C}\right)$ and $\delta_{C}=\operatorname{res}_{F \mid F_{C}}(\delta)$ by Corollary 5.6, hence $\operatorname{ind}(\delta)=\operatorname{ind}\left(\delta_{C_{1}}\right)$ and $\delta_{C_{1}}=\operatorname{res}_{F \mid F_{C_{1}}}(\delta)$ by definition of index and Brauer class over $F_{C}$. Let $D$ be the $F$-division algebra associated to $\delta$. Since $\operatorname{ind}(\delta)=\operatorname{ind}\left(\delta_{C_{1}}\right)$ $D \otimes_{F} F_{C_{1}}$ is isomorphic to the $F_{C_{1}}$-division algebra $D_{F_{C_{1}}}$ associated to $\delta_{C_{1}}$. If $D$ is decomposable, then $D \simeq D_{1} \otimes_{F} D_{2}$ for nontrivial $F$-division algebras $D_{1}$ and $D_{2}$, hence $D_{F_{C_{1}}}=\left(D_{1}\right)_{F_{C_{1}}} \otimes_{F_{C_{1}}}\left(D_{2}\right)_{F_{C_{1}}}$ is a decomposition of $D_{F_{C_{1}}}$. Since $D_{F_{C_{1}}}$ is a division algebra, both factors $\left(D_{i}\right)_{F_{C_{1}}}$ are nontrivial, hence $D_{F_{C_{1}}}$ is decomposable, a contradiction. We conclude $D$ is indecomposable.

\section{REFERENCES}

[1] S. A. Amitsur, On central division algebras, Israel J. Math. 12 (1972), 408-420. MR.0318216 $(47$ \#6763)

[2] S. A. Amitsur, Division algebras. A survey, Algebraists' homage: papers in ring theory and related topics (New Haven, Conn., 1981), Contemp. Math., vol. 13, Amer. Math. Soc., Providence, R.I., 1982, pp. 3-26. MR685935 (84b:16021)

[3] S. A. Amitsur, L. H. Rowen, and J.-P. Tignol, Division algebras of degree 4 and 8 with involution, Israel J. Math. 33 (1979), no. 2, 133-148, DOI 10.1007/BF02760554. MR571249 (81h:16029)

[4] M. F. Atiyah and I. G. Macdonald, Introduction to commutative algebra, Addison-Wesley Publishing Co., Reading, Mass.-London-Don Mills, Ont., 1969. MR0242802 (39 \#4129)

[5] Asher Auel, Eric Brussel, Skip Garibaldi, and Uzi Vishne, Open problems on central simple algebras, Transform. Groups 16 (2011), no. 1, 219-264, DOI 10.1007/s00031-011-9119-8. MR2785502 (2012e:16050)

[6] Eric Brussel, Noncrossed products and nonabelian crossed products over $\mathbf{Q}(t)$ and $\mathbf{Q}((t))$, Amer. J. Math. 117 (1995), no. 2, 377-393, DOI 10.2307/2374919. MR1323680(96a:16014)

[7] Eric S. Brussel, Decomposability and embeddability of discretely Henselian division algebras. part A, Israel J. Math. 96 (1996), no. part A, 141-183, DOI 10.1007/BF02785537. MR:1432730 (97m:16030)

[8] Eric S. Brussel, Noncrossed products over $k_{\mathfrak{p}}(t)$, Trans. Amer. Math. Soc. 353 (2001), no. 5, 2115-2129 (electronic), DOI 10.1090/S0002-9947-00-02626-X. MR.1813610 (2001j:16022)

[9] Eric Brussel, On Saltman's p-adic curves papers, Quadratic forms, linear algebraic groups, and cohomology, Dev. Math., vol. 18, Springer, New York, 2010, pp. 13-39, DOI 10.1007/9781-4419-6211-9_2. MR2648718 (2011k:16041) 
[10] E. Brussel, K. McKinnie, and E. Tengan, Indecomposable and noncrossed product division algebras over function fields of smooth p-adic curves, Adv. Math. 226 (2011), no. 5, 43164337, DOI 10.1016/j.aim.2010.12.005. MR2770451 (2012g:14033)

[11] E. Brussel and E. Tengan, Division algebras of prime period $\ell \neq p$ over function fields of p-adic curves, Israel J. Math. (to appear).

[12] J.-L. Colliot-Thélène, Birational invariants, purity and the Gersten conjecture, division algebras (Santa Barbara, CA, 1992), Proc. Sympos. Pure Math., vol. 58, Amer. Math. Soc., Providence, RI, 1995, pp. 1-64. MR.1327280 (96c:14016)

[13] Eberhard Freitag and Reinhardt Kiehl, Etale cohomology and the Weil conjecture, Ergebnisse der Mathematik und ihrer Grenzgebiete (3) [Results in Mathematics and Related Areas (3)], vol. 13, Springer-Verlag, Berlin, 1988. Translated from the German by Betty S. Waterhouse and William C. Waterhouse; With an historical introduction by J. A. Dieudonné. MR.926276 (89f:14017)

[14] László Fuchs, Infinite abelian groups. Vol. I, Pure and Applied Mathematics, Vol. 36, Academic Press, New York, 1970. MR0255673 (41 \#333)

[15] Kazuhiro Fujiwara, A proof of the absolute purity conjecture (after Gabber), Algebraic geometry 2000, Azumino (Hotaka), Adv. Stud. Pure Math., vol. 36, Math. Soc. Japan, Tokyo, 2002, pp. 153-183. MR 1971516 (2004d:14015)

[16] Skip Garibaldi, Alexander Merkurjev, and Jean-Pierre Serre, Cohomological invariants in Galois cohomology, University Lecture Series, vol. 28, American Mathematical Society, Providence, RI, 2003. MR1999383 (2004f:11034)

[17] A. Grothendieck, Éléments de géométrie algébrique. I. Le langage des schémas, Inst. Hautes Études Sci. Publ. Math. 4 (1960), 228. MR0217083 (36 \#177a)

[18] A. Grothendieck, Éléments de géométrie algébrique. IV. Étude locale des schémas et des morphismes de schémas. II (French), Inst. Hautes Études Sci. Publ. Math. 24 (1965), 231. MR0199181 (33 \#7330)

[19] A. Grothendieck, Éléments de géométrie algébrique. IV. Étude locale des schémas et des morphismes de schémas. III, Inst. Hautes Études Sci. Publ. Math. 28 (1966), 255. MR0217086 (36 \#178)

[20] A. Grothendieck, Éléments de géométrie algébrique. IV. Étude locale des schémas et des morphismes de schémas IV (French), Inst. Hautes Études Sci. Publ. Math. 32 (1967), 361. MR0238860 (39 \#220)

[21] A. Grothendieck, Revêtements étales et groupe fondamental (SGA 1) (French), Documents Mathématiques (Paris) [Mathematical Documents (Paris)], 3, Société Mathématique de France, Paris, 2003. Séminaire de géométrie algébrique du Bois Marie 1960-61. [Algebraic Geometry Seminar of Bois Marie 1960-61]; Directed by A. Grothendieck; With two papers by M. Raynaud; Updated and annotated reprint of the 1971 original [Lecture Notes in Math., 224, Springer, Berlin; MR0354651 (50 \#7129)]. MR2017446 (2004g:14017)

[22] Alexander Grothendieck and Jacob P. Murre, The tame fundamental group of a formal neighbourhood of a divisor with normal crossings on a scheme, Lecture Notes in Mathematics, Vol. 208, Springer-Verlag, Berlin, 1971. MR0316453 (47 \#5000)

[23] David Harbater, Julia Hartmann, and Daniel Krashen, Applications of patching to quadratic forms and central simple algebras, Invent. Math. 178 (2009), no. 2, 231-263, DOI 10.1007/s00222-009-0195-5. MR2545681 (2010j:11058)

[24] David Harbater, Julia Hartmann, and Daniel Krashen, Patching subfields of division algebras, Trans. Amer. Math. Soc. 363 (2011), no. 6, 3335-3349, DOI 10.1090/S0002-9947-2010-052298. MR2775810 (2012c:12008)

[25] Bill Jacob, Indecomposable division algebras of prime exponent, J. Reine Angew. Math. 413 (1991), 181-197, DOI 10.1515/crll.1991.413.181. MR.1089801 (91m:12002)

[26] N. Karpenko, Chow groups of quadrics and index reduction formula, Nova J. Algebra Geom. 3 (1995), no. 4, 357-379. MR1341100 (96e:14003)

[27] Nikita A. Karpenko, Codimension 2 cycles on Severi-Brauer varieties, K-Theory 13 (1998), no. 4, 305-330, DOI 10.1023/A:1007705720373. MR1615533(99b:16030)

[28] Kazuya Kato, A Hasse principle for two-dimensional global fields, J. Reine Angew. Math. 366 (1986), 142-183, DOI 10.1515/crll.1986.366.142. With an appendix by Jean-Louis ColliotThélène. MR833016 (88b:11036) 
[29] Serge Lang, Algebra, 3rd ed., Graduate Texts in Mathematics, vol. 211, Springer-Verlag, New York, 2002. MR 1878556 (2003e:00003)

[30] Max Lieblich, Period and index in the Brauer group of an arithmetic surface, J. Reine Angew. Math. 659 (2011), 1-41, DOI 10.1515/CRELLE.2011.059. With an appendix by Daniel Krashen. MR.2837009

[31] Qing Liu, Algebraic geometry and arithmetic curves, Oxford Graduate Texts in Mathematics, vol. 6, Oxford University Press, Oxford, 2002. Translated from the French by Reinie Erné; Oxford Science Publications. MR1917232 (2003g:14001)

[32] Hideyuki Matsumura, Commutative ring theory, 2nd ed., Cambridge Studies in Advanced Mathematics, vol. 8, Cambridge University Press, Cambridge, 1989. Translated from the Japanese by M. Reid. MR.1011461 (90i:13001)

[33] James S. Milne, Étale cohomology, Princeton Mathematical Series, vol. 33, Princeton University Press, Princeton, N.J., 1980. MR559531 (81j:14002)

[34] Raman Parimala and V. Suresh, The u-invariant of the function fields of p-adic curves, Ann. of Math. (2) 172 (2010), no. 2, 1391-1405, DOI 10.4007/annals.2010.172.1397. MR2680494 (2011g:11074)

[35] Wayne Raskind, Abelian class field theory of arithmetic schemes, division algebras (Santa Barbara, CA, 1992), Proc. Sympos. Pure Math., vol. 58, Amer. Math. Soc., Providence, RI, 1995, pp. 85-187. MR1327282(96b:11089)

[36] Shuji Saito, Class field theory for curves over local fields, J. Number Theory 21 (1985), no. 1, 44-80, DOI 10.1016/0022-314X(85)90011-3. MR804915 (87g:11075)

[37] David J. Saltman, Indecomposable division algebras, Comm. Algebra 7 (1979), no. 8, 791-817, DOI 10.1080/00927877908822376. MR529494 (80g:16021)

[38] David J. Saltman, Finite-dimensional division algebras, Azumaya algebras, actions, and modules (Bloomington, IN, 1990), Contemp. Math., vol. 124, Amer. Math. Soc., Providence, RI, 1992, pp. 203-214, DOI 10.1090/conm/124/1144037. MR 1144037 (93a:16014)

[39] David J. Saltman, Division algebras over p-adic curves, J. Ramanujan Math. Soc. 12 (1997), no. 1, 25-47. MR1462850 (98d:16032)

[40] David J. Saltman, Correction to: "Division algebras over p-adic curves" [J. Ramanujan Math. Soc. 12 (1997), no. 1, 25-47; MR1462850 (98d:16032)], J. Ramanujan Math. Soc. 13 (1998), no. 2, 125-129. MR.1666362 (99k:16036)

[41] David J. Saltman, Cyclic algebras over p-adic curves, J. Algebra 314 (2007), no. 2, 817-843, DOI 10.1016/j.jalgebra.2007.03.003. MR2344586 (2008i:16018)

[42] Venapally Suresh, Bounding the symbol length in the Galois cohomology of function fields of p-adic curves, Comment. Math. Helv. 85 (2010), no. 2, 337-346, DOI 10.4171/CMH/198. MR2595182 (2011b:12010)

Department of Mathematics, California Polytechnic State University, San Luis Obispo, California 93407

E-mail address: ebrussel@calpoly.edu

Instituto de Ciências Matemáticas e de ComputaÇão, Universidade de São Paulo, São Carlos, São Paulo, Brazil

E-mail address: etengan@icmc.usp.br 